\title{
Ground-based observation of ZZ Ceti stars and the discovery of four new variables
}

\author{
Alejandra D. Romero ${ }^{\oplus},{ }^{1 \star}$ L. Antunes Amaral, ${ }^{1}$ T. Klippel, ${ }^{1}$ D. Sanmartim ${ }^{\oplus},{ }^{2}$ \\ L. Fraga,${ }^{3}$ G. Ourique, ${ }^{1}$ I. Pelisoli ${ }^{\oplus},{ }^{4}$ G. R. Lauffer ${ }^{\odot},{ }^{1}$ S. O. Kepler ${ }^{\oplus 1}$ and D. Koester ${ }^{5}$ \\ ${ }^{1}$ Physics Institute, Universidade Federal do Rio Grande do Sul, Av. Bento Gonçalves 9500, Porto Alegre, RS-91501-970, Brazil \\ ${ }^{2}$ Gemini Observatory, c/o AURA - Casilla 603, La Serena, Chile \\ ${ }^{3}$ Laboratório Nacional de Astrofísica LNA/MCTIC, 37504-364 Itajubá, MG, Brazil \\ ${ }^{4}$ Institut für Physik und Astronomie, Universitätsstandort Golm, Karl-Liebknecht-Str 24/25, D-14467 Potsdam, Germany \\ ${ }^{5}$ Institut für Theoretische Physik und Astrophysik, Universität Kiel, D-24098 Kiel, Germany
}

Accepted 2019 September 10. Received 2019 September 10; in original form 2019 July 9

\begin{abstract}
We perform ground-based photometric observations of 22 DA white dwarf stars, 10 already known ZZ Cetis and 12 candidates with atmospheric parameters inside the classical instability strip. We report on the discovery of four new variable DA white dwarf stars. Two objects are near the middle of the instability strip, SDSS J082804.63+094956.6 and SDSS J094929.09+101918.8, and two red edge pulsators, GD 195 and L495-82. In addition, we classified four objects as possible variables, since evidence of variability was detected in the light curve, but the signal-to-noise ratio was not sufficient to establish a definite detection. Follow-up observations were performed for 10 known ZZ Ceti stars to verify period stability and search for new periodicities. For each confirmed variable, we perform a detailed asteroseismological fit and compare the structural parameters obtained from the best-fitting models with those obtained from spectroscopy and photometry from Gaia. Finally we present a study of the asteroseismological properties of a sample of 91 ZZ Ceti stars.
\end{abstract}

Key words: stars: evolution -stars: variables: general - white dwarf.

\section{INTRODUCTION}

ZZ Ceti stars are white dwarf stars with hydrogen-dominated atmospheres (DA) that show periodic variability. The instability strip of the ZZ Ceti is between 13000 and $10000 \mathrm{~K}$, depending on stellar mass (Hermes et al. 2017; Kepler \& Romero 2017). Their photometric variations are due to surface temperature changes explained by spheroidal non-radial g-mode pulsations with low harmonic degree $(\ell \leq 2)$ and periods between 70 and $2000 \mathrm{~s}$, with amplitude variations up to $0.3 \mathrm{mag}$. To date, there are $\sim 250 \mathrm{ZZ}$ Cetis known (see Bognar \& Sodor 2016; Córsico et al. 2019).

The driving mechanism for the excitation of the pulsations is the $\kappa-\gamma$ mechanism acting on the hydrogen partial ionization zone (Dolez \& Vauclair 1981; Winget et al. 1982) for the blue edge of the instability strip. The convective driving mechanism (Brickhill 1991; Goldreich \& Wu 1999) is considered to be dominant once a thick convective zone has developed in the outer layers.

The ZZ Cetis can be classified into three groups, depending on the effective temperature and the stellar mass (Clemens 1993; Mukadam et al. 2006). The hot $\mathrm{ZZ}$ Cetis, which define the blue edge of the instability strip, exhibit a few modes with short periods (<350 s) and small amplitudes (1.5-20 mma). The pulse shape is sinusoidal or sawtooth shaped and is stable for decades. On the opposite side of the instability strip are the cool DAV stars, showing several long periods (up to $1500 \mathrm{~s}$ ), with large amplitudes (40-110 mma), and non-sinusoidal light curves that change dramatically from season to season due to mode interference. Mukadam et al. (2006) suggested introducing a third class, the intermediate ZZ Cetis, with mixed characteristics from hot and cool ZZ Cetis.

Up until the Sloan Digital Sky Survey (SDSS), less than $~ 50$ ZZ Cetis were known (e.g. Fontaine \& Brassard 2008), all with magnitudes $V<16$. The number of known DA white dwarfs, and thus of DA pulsators, dramatically increased to $\sim 170$ members with the SDSS and the effort of several authors conducting groundbased observations (Mukadam et al. 2004; Mullally et al. 2005; Kepler et al. 2005, 2012; Castanheira et al. 2006, 2007; Castanheira et al. 2010, 2013; Romero et al. 2013).

The list was enlarged with the discovery of pulsating white dwarfs stars within the Kepler spacecraft field, ${ }^{1}$ thus opening a new avenue for white dwarf asteroseismology based on observa- 
tions from space. This kind of data do not have the usual gaps due to daylight and also can cover months. However, the data reduction is quite challenging since a collection of instrumental frequencies, in the same range as those for known pulsators, needs to be subtracted from the data (Gilliland et al. 2010; Baran 2013). The first ZZ Ceti with published data was GD 1212 (Hermes et al. 2014), already classified as variable by Gianninas, Bergeron \& Fontaine (2006), while the ZZ Ceti star observed the longest by the Kepler spacecraft was KIC 4552982, with data spanning more than 1.5 yr. In particular, KIC 4552982 was the first ZZ Ceti to show energetic outbursts that increase the relative flux of the star by 2 percent-17 percent (Bell et al. 2015). Hermes et al. (2017) presented photometry and spectroscopy for 27 DAVs observed by the Kepler spacecraft, including six DAVs known at the time. They used this homogeneously analysed sample to study the white dwarfs rotation as a function of mass.

Data of similar quality to that provided by Kepler will be obtained by the Transiting Exoplanet Survey Satellite ${ }^{2}$ (TESS), launched in 2018 April, which will perform a wide-field survey for planets that transit bright host stars (Ricker et al. 2014). Compact pulsators, as white dwarfs and subdwarf stars, will be studied with TESS since 2min cadence photometry is available (Bell et al. 2019). The activities related to compact pulsators are coordinated by the TESS Compact Pulsators Working Group (WG\#8).

Time-resolved ground-based observations of variable white dwarf stars can help to increase the number of ZZ Ceti stars, and also other types of compact pulsators, to better understand the properties of ZZ Cetis and DA white dwarf stars in general. They can also function as a complement of space-based surveys, given that in some cases the resolution necessary to detect pulsations in variable DA white dwarfs is restricted to bright objects, especially for the ones near the blue edge of the instability strip. In addition, most of the known ZZ Ceti stars have pulsation periods only from the discovery observations. Follow-up observations of known pulsators can uncover new periodicities, improving the seismological studies. Finally, the stability of the pulsation modes, in amplitude and period, can carry information on the inner structure of the star as well (Montgomery et al. 2010).

In this paper, we carry out time-series photometry observations of 22 DA white dwarfs. We performed follow-up observations on 10 known ZZ Ceti stars, and observe 12 ZZ Ceti candidates selected from spectroscopic parameters. For each object with confirmed variability, we perform a detailed asteroseismological fit by employing an expanded version of the grid of full evolutionary DA white dwarf models presented in Romero et al. (2017).

This paper is organized as follows: we present our sample selection in Section 2, describe the data reduction in Section 3, and present the observational results in Section 4. In Section 5 we present our asteroseismological fits for the objects that show photometric variability. We present photometric determinations of effective temperature and stellar mass using Gaia magnitudes and parallax in Section 6. In Section 7 we present a study of the asteroseismological properties of a sample of $77 \mathrm{ZZ}$ Ceti stars, including the ones analysed in this work, that have been subject of an asteroseismological study. We conclude in Section 8 by summarizing our findings.

${ }^{2}$ https://heasarc.gsfc.nasa.gov/docs/tess/

\section{SAMPLE SELECTION}

We selected a list of targets from a sample of DA white dwarfs from the catalogues presented by Kleinman et al. (2013) and Kepler et al. $(2016,2019)$ from SDSS. We choose those objects with spectroscopic effective temperature and surface gravity within the instability strip of the $\mathrm{ZZ}$ Cetis. In addition, we consider a sample of objects from the list of white dwarfs presented by Bédard, Bergeron \& Fontaine (2017), also with spectroscopic atmospheric parameters within the instability strip, that were not classified as variable white dwarfs. Finally, we selected a sample of known ZZ Ceti stars, most of them with published data corresponding only to the discovery paper, and no follow-up observations. A list of the objects observed in this work is presented in Table 1, where we list the spectroscopic effective temperature and surface gravity with and without 3D convection correction from Tremblay et al. (2013). The stellar mass values were estimated by linear interpolation of the evolutionary tracks (Romero et al. 2012, 2013) in the $\log g-T_{\text {eff }}$ diagram, given the values of $\log g$ and effective temperature from Table 1. We consider the spectroscopic values with 3D convection correction and the evolutionary sequences characterized by canonical hydrogen envelopes, i.e. those with the thickest value as predicted by single stellar evolution allowed by nuclear burning (see Romero et al. 2019, for details). The location of all observed objects on the $T_{\text {eff }}-\log g$ plane is depicted in Fig. 1. The $\sim 250 \mathrm{ZZ}$ Cetis stars known to date are depicted in this figure, and were extracted from the works of Bognar \& Sodor (2016) (blue up-triangle), $\mathrm{Su}$ et al. (2017) (green left-triangle), Hermes et al. (2017) (red down-triangle), Bell et al. (2017) (violet right-triangle), and Rowan et al. (2019) (magenta square). The values for effective temperature and surface gravity were corrected by 3D convection for all objects (Córsico et al. 2019). The objects observed in this work are classified as candidates and known and depicted with full and hollow black circles, respectively.

\section{OBSERVATIONS AND DATA REDUCTION}

We employed Goodman image mode on the 4.1-m Southern Astrophysical Research (SOAR) Telescope from 2015 to 2019. We used read out mode $200 \mathrm{~Hz}$ ATTN2 with the CCD binned $2 \times 2$. All observations were obtained with a red blocking filter S8612. The integration times varies from 10 to $60 \mathrm{~s}$, depending on the magnitude of the object and the weather conditions.

In addition, we used the IxON camera on the 1.6-m Perkin Elmer Telescope at the Pico dos Dias Observatory during 2016, 2017, and 2018. We also used a red blocking filter BG40. The integration times varies from 20 to $45 \mathrm{~s}$, depending on the magnitude of the object. The journal of observations is shown in Table 2.

We reduced the data with the software IRAF, and perform aperture photometry with DAOFOT. We extracted light curves of all bright stars that were observed simultaneously in the field. Then, we divided the light curve of the target star by the light curves of all comparison stars to minimize effects of sky and transparency fluctuations. To look for periodicities in the light curves, we calculate the Fourier transform (FT) using the software PERIOD04 (Lenz \& Breger 2004). We accepted a frequency peak as significant if its amplitude exceeds an adopted significance threshold. In this work, we adopted a $4<\mathrm{A}>$ significance criterion, where $<\mathrm{A}>$ is the mean amplitude of the FT, corresponding to a probability of the peak being due to noise smaller than 1 in 1000 (Kepler 1993). We then use the process of pre-whitening the light curve by subtracting 
Table 1. Atmospheric parameters for the sample stars (columns 2 and 3), obtained from spectroscopy, and the stellar mass (column 4). The values corrected using the 3D convection correction (Córsico et al. 2019) are listed in columns 5 and 6, and the resulting stellar mass is listed in column 7. Column 8 lists the references: (1) Gianninas, Bergeron \& Ruiz (2011), (2) Kleinman et al. (2013), (3) Bédard et al. (2017), and (4) Kepler et al. (2019).

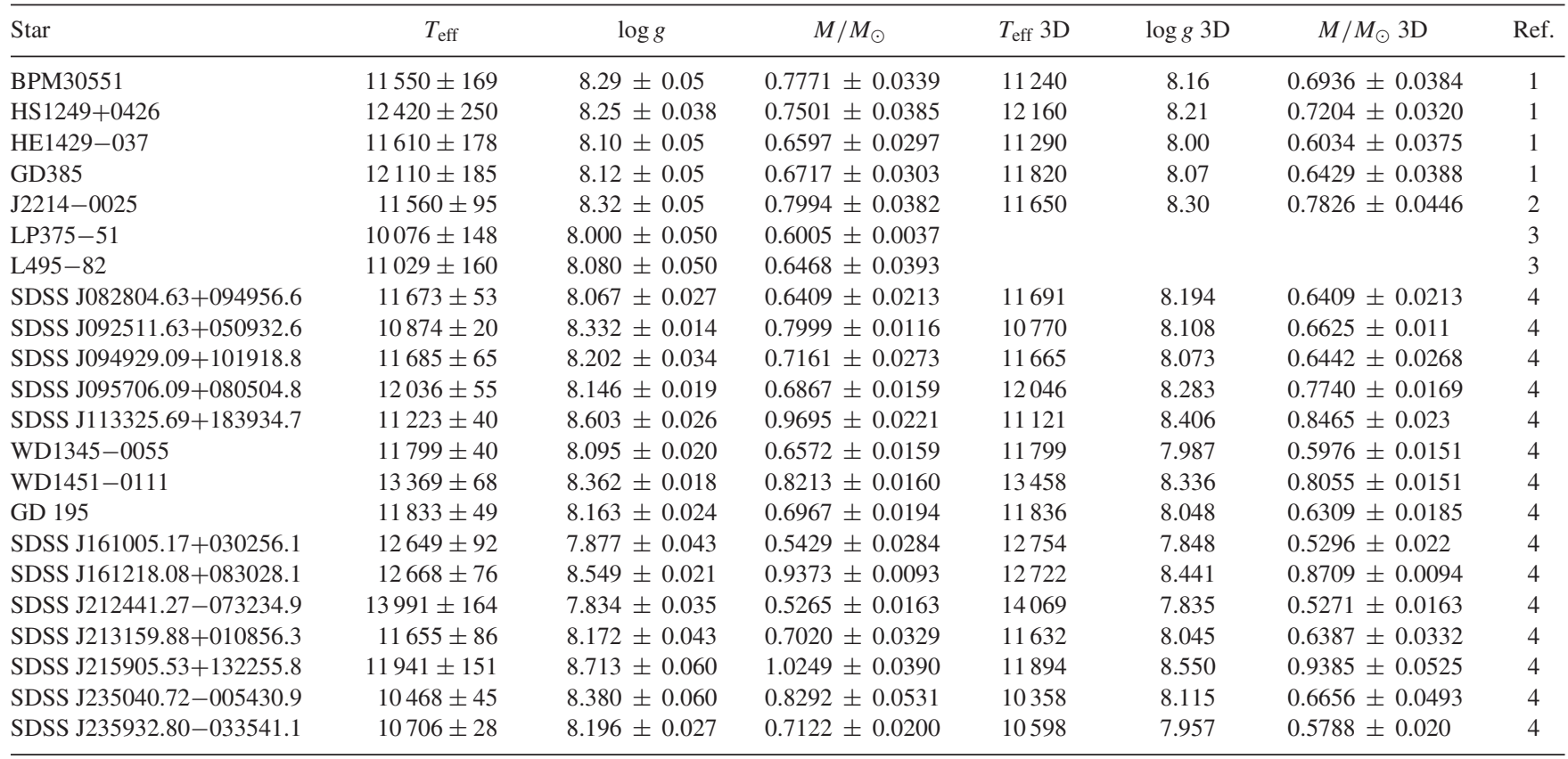

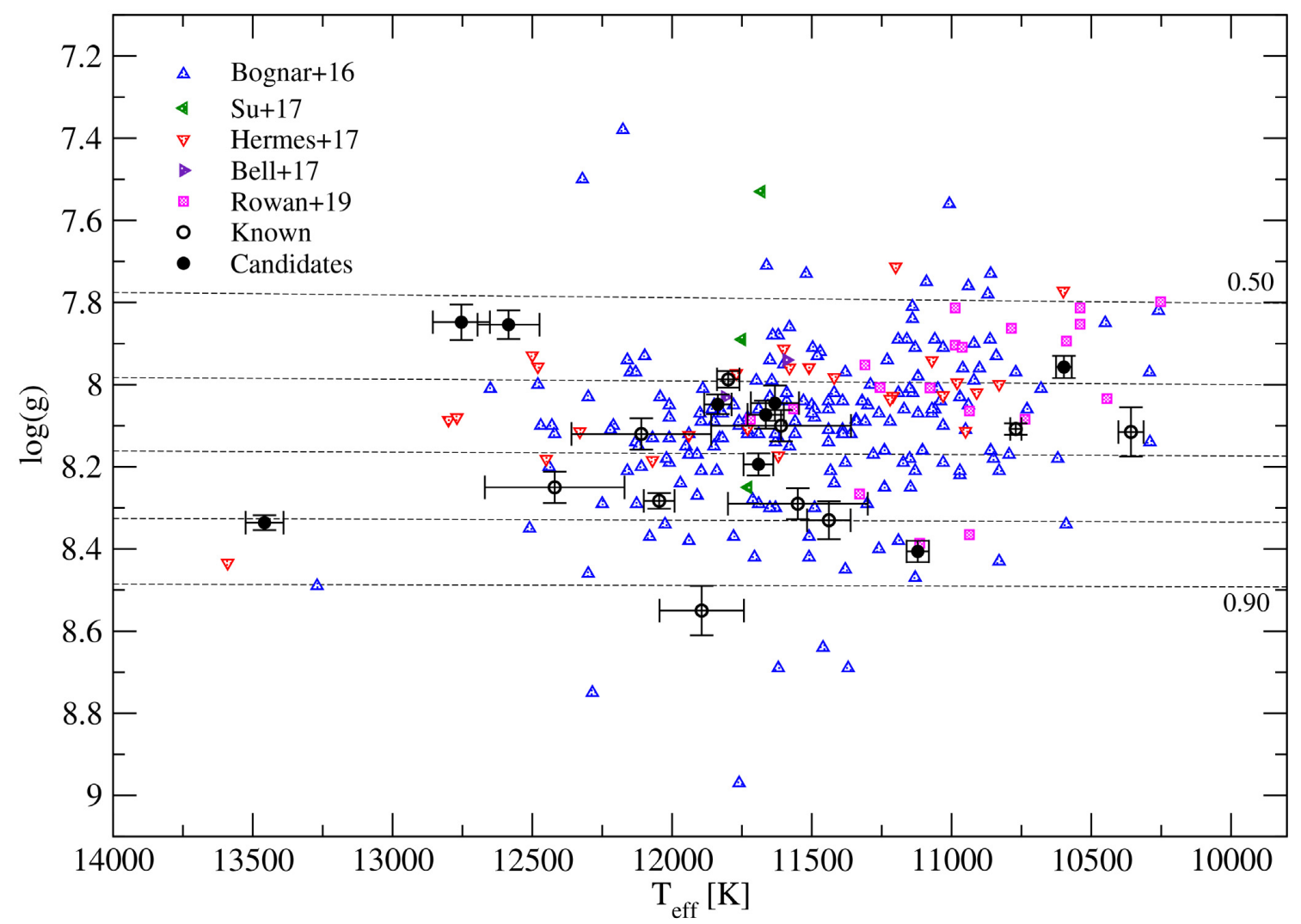

Figure 1. Distribution of ZZ Ceti stars on the $T_{\text {eff }}-\log g$ plane. The coloured symbols correspond to the ZZ Ceti stars known to date, extracted from Bognar \& Sodor (2016) (blue up-triangle), Su et al. (2017) (green left-triangle), Hermes et al. (2017) (red down-triangle), Bell et al. (2017) (violet right-triangle), and Rowan et al. (2019) (magenta square). The objects observed in this work are depicted with black circles, identified as candidates (full circle) and known variables (hollow circles). We include evolutionary tracks (dashed lines) with stellar masses between 0.5 and $0.9 \mathrm{M}_{\odot}$ from top to bottom (Romero et al. 2019). 
Table 2. Journal of observations for the objects observed. $\Delta t$ is the total length of each observing run and $t_{\exp }$ is the integration time of each exposure.

\begin{tabular}{|c|c|c|c|c|c|c|c|}
\hline Star & RA & Dec. & $g$ & Telescope & Run start (UT) & $t_{\exp }(\mathrm{s})$ & $\Delta t(\mathrm{~h})$ \\
\hline \multicolumn{8}{|l|}{ Known variables } \\
\hline \multirow[t]{2}{*}{ BPM 30551} & 010653.68 & -460853.73 & 15.47 & SOAR & $2016-08-23$ 08:35:52.83 & 10 & 1.55 \\
\hline & & & & OPD & 2016-08-30 06:20:21.12 & 10 & 2.08 \\
\hline \multirow[t]{2}{*}{ SDSS J092511.63+050932.6 } & 092511.63 & +050932.6 & 15.20 & OPD & 2016-04-17 22:41:16.91 & 35 & 2.47 \\
\hline & & & & OPD & 2016-04-17 00:19:40.72 & 35 & 1.00 \\
\hline \multirow[t]{2}{*}{ HS $1249+0426$} & 125215.19 & +041052.9 & 16.04 & OPD & 2016-04-16 03:46:00.40 & 30 & 1.90 \\
\hline & & & & OPD & 2016-04-18 03:27:02.32 & 45 & 2.03 \\
\hline WD1345-0055 & 134550.92 & -005536.4 & 16.78 & OPD & 2016-04-17 03:56:59.02 & 15 & 2.50 \\
\hline HE1429-037 & 143203.19 & -035638.2 & 16.03 & OPD & 2017-04-17 03:28:43.74 & 30 & 1.87 \\
\hline SDSS J161218.08+083028.1 & 161218.08 & +083028.1 & 17.75 & SOAR & 2014-07-02 00:11:16.35 & 30 & 1.87 \\
\hline GD 385 & 195227.88 & +250929.10 & 16.63 & OPD & 2016-08-29 23:08:02.08 & 10 & 3.47 \\
\hline SDSS J215905.53+132255.8 & 215905.53 & +132255.8 & 18.99 & SOAR & 2016-08-22 01:38:56.16 & 30 & 2.00 \\
\hline SDSS J221458.37-002511.9 & 221458.37 & -002511.91 & 17.92 & OPD & 2016-08-29 01:58:31.27 & 30 & 4.12 \\
\hline SDSS J235040.72-005430.9 & 235040.72 & -005430.87 & 18.12 & SOAR & 2016-08-22 04:17:18.89 & 15 & 2.00 \\
\hline \multicolumn{8}{|l|}{ Variable candidates } \\
\hline \multirow[t]{2}{*}{ SDSS J082804.63+049456.6 } & 082804.63 & +094956.66 & 17.71 & SOAR & 2016-12-24 03:57:54.27 & 15 & 2.06 \\
\hline & & & & SOAR & 2016-12-27 04:16:49.31 & 15 & 4.19 \\
\hline \multirow[t]{3}{*}{ SDSS J094929.09+101918.85 } & 094929.09 & +101918.85 & 17.58 & SOAR & 2016-12-24 06:09:23.07 & 15 & 2.07 \\
\hline & & & & SOAR & 2017-01-29 07:16:50.90 & 15 & 1.29 \\
\hline & & & & SOAR & 2015-03-19 00:15:51.21 & 30 & 4.26 \\
\hline \multirow[t]{3}{*}{ SDSS J095703.09+080504.8 } & 095703.09 & +080504.85 & 17.70 & OPD & 2017-04-15 01:58:40.26 & 40 & 1.16 \\
\hline & & & & OPD & 2017-04-16 22:09:13.03 & 40 & 4.50 \\
\hline & & & & SOAR & 2017-01-29 03:45:34.29 & 20 & 3.11 \\
\hline \multirow[t]{2}{*}{ SDSS J113325.09+183934.7 } & 113325.69 & +183934.75 & 17.59 & OPD & 2017-04-15 00:37:22.27 & 50 & 2.65 \\
\hline & & & & OPD & 2018-05-10 22:00:03.75 & 20 & 4.14 \\
\hline LP $375-51$ & 115020.17 & +251832.76 & 15.70 & OPD & 2018-05-11 23:44:21.80 & 30 & 2.66 \\
\hline WD1454-0111 & 145436.08 & -011152.5 & 17.34 & OPD & 2016-04-15 06:14:45.5 & 30 & 2.22 \\
\hline \multirow[t]{4}{*}{ GD 195} & 160746.21 & +173720.76 & 16.63 & OPD & 2016-04-18 06:09:35.46 & 50 & 2.33 \\
\hline & & & & OPD & 2016-04-18 05:51:50.89 & 40 & 2.65 \\
\hline & & & & OPD & 2016-04-17 07:04:47.12 & 40 & 1.44 \\
\hline & & & & OPD & 2017-04-16 05:16:58.56 & 20 & 3.00 \\
\hline SDSS J161005.17+030256.1 & 161005.17 & +030256.07 & 18.55 & SOAR & 2017-08-06 23:08:09.69 & 15 & 3.00 \\
\hline $\mathrm{L} 495-82$ & 204349.2 & -390318.2 & 13.76 & OPD & 2018-05-12 05:11:34.94 & 10 & 2.85 \\
\hline \multirow[t]{2}{*}{ SDSS J212441.27-073234.9 } & 212441.27 & -073234.93 & 18.47 & SOAR & 2019-05-21 07:31:23.14 & 60 & 2.44 \\
\hline & & & & SOAR & 2019-05-22 07:32:59.99 & 22 & 2.60 \\
\hline \multirow[t]{2}{*}{ SDSS J213159.88+010856.3 } & 233159.88 & +010856.26 & 18.40 & SOAR & 2017-08-07 02:44:00.29 & 30 & 1.88 \\
\hline & & & & SOAR & 2017-07-13 07:08:01.08 & 30 & 2.40 \\
\hline SDSS J235932.80-033541.1 & 235932.80 & -033541.07 & 17.91 & SOAR & 2017-07-22 09:02:34.37 & 10 & 1.58 \\
\hline
\end{tabular}

out of the data a sinusoid with the same frequency, amplitude, and phase of highest peak and then computing the FT for the residuals. We redo this process until we have no new significant signals. The objects classified as candidates were observed for a minimum of three hours in total to confirm variation. As a result, we find four new ZZ Ceti stars among the candidates. We also discovered new periods for some known variables.

\section{OBSERVATIONAL RESULTS}

In this section, we present the results from the observations for the 22 objects observed for this work. We found four new ZZ Ceti stars and four possible new variables. For the known pulsators, we recovered most of the periods from the literature and detected new modes. From the FT we were not able to detect any multiplets to extract information on the harmonic degree. Finally, in the case of the rich pulsators, we looked for linear combinations among the detected periodicities, to select those periods corresponding to real pulsation modes. We detail the results from the observations below.

\subsection{New ZZ Cetis}

From the observed sample, we found four new ZZ Ceti stars: SDSS J082804.63+094956.6, SDSS J094929.09+101918.8, GD 195, and L495-82. We present the results for each object below. The light curves and FT for each object are depicted in Figs 2, 3, 4, and 5, respectively, while the list of observed frequencies, periods, and amplitudes is presented in Table $3 .^{3}$

\subsubsection{SDSS J082804.63+094956.6}

The star J0828+0949 was selected as a candidate from the SDSS catalogue presented by Kepler et al. (2016). It was observed in two nights for a total of six hours with the SOAR telescope. In Fig. 2 we show the light curve for the four-hour run (top panel), and the FT corresponding to all observation nights (bottom panel), where

\footnotetext{
${ }^{3}$ The uncertainties in the frequencies and their amplitude are listed in Table A1.
} 

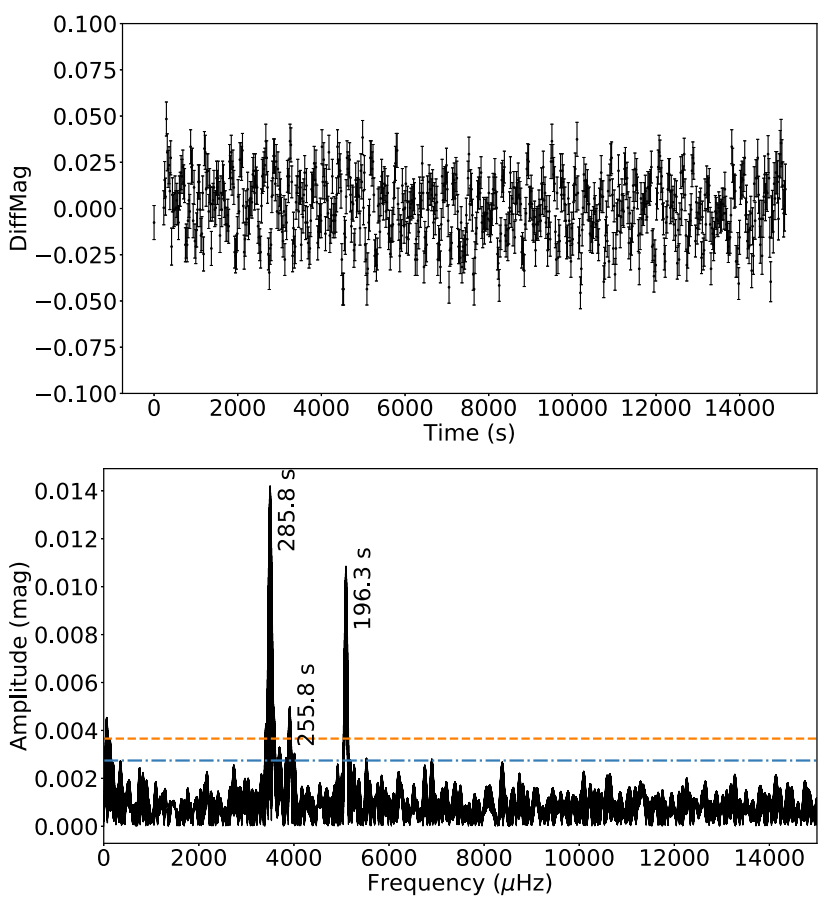

Figure 2. Light curve (top panel) and FT (bottom panel) for the object SDSS J082804.63+094956.6. The light curve corresponds to the four-hour run, while for the FT we consider the two observation nights. The orange dashed (blue dot-dashed) line corresponds to the $4 \sigma(3 \sigma)$ detection limit.
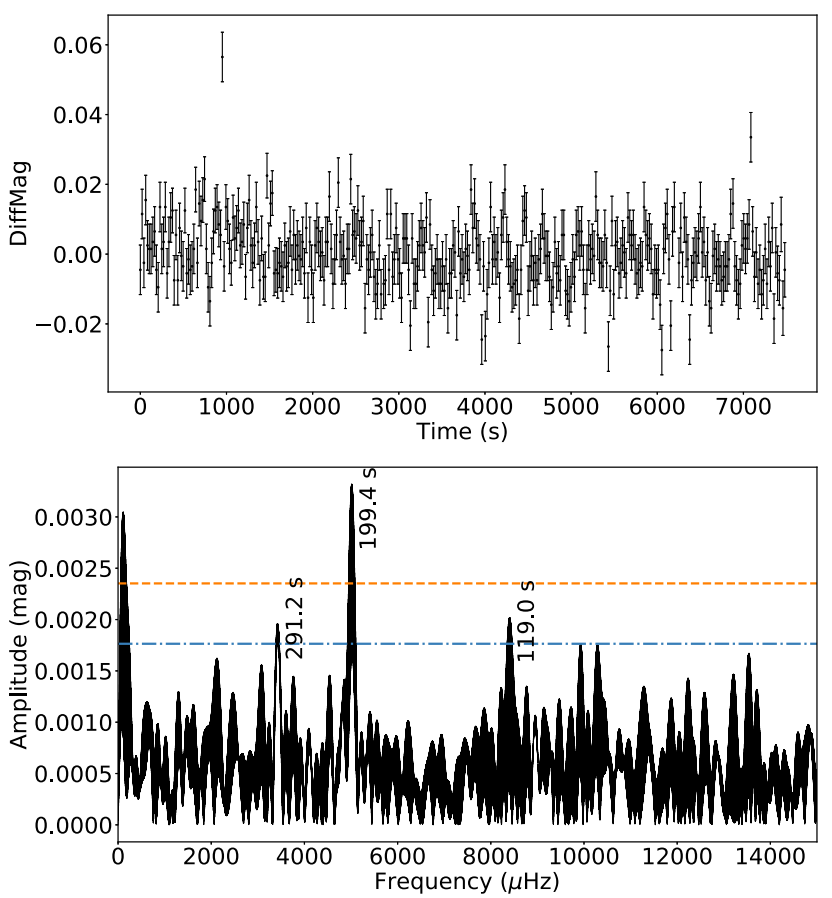

Figure 3. Light curve (top panel) and FT (bottom panel) for the star SDSS J094929.09+101918.8. The light curve corresponds to the $2.07 \mathrm{~h}$ observation run. The FT is the result from the sum of both nights. The orange dashed (blue dot-dashed) line corresponds to the $4 \sigma(3 \sigma)$ detection limit.
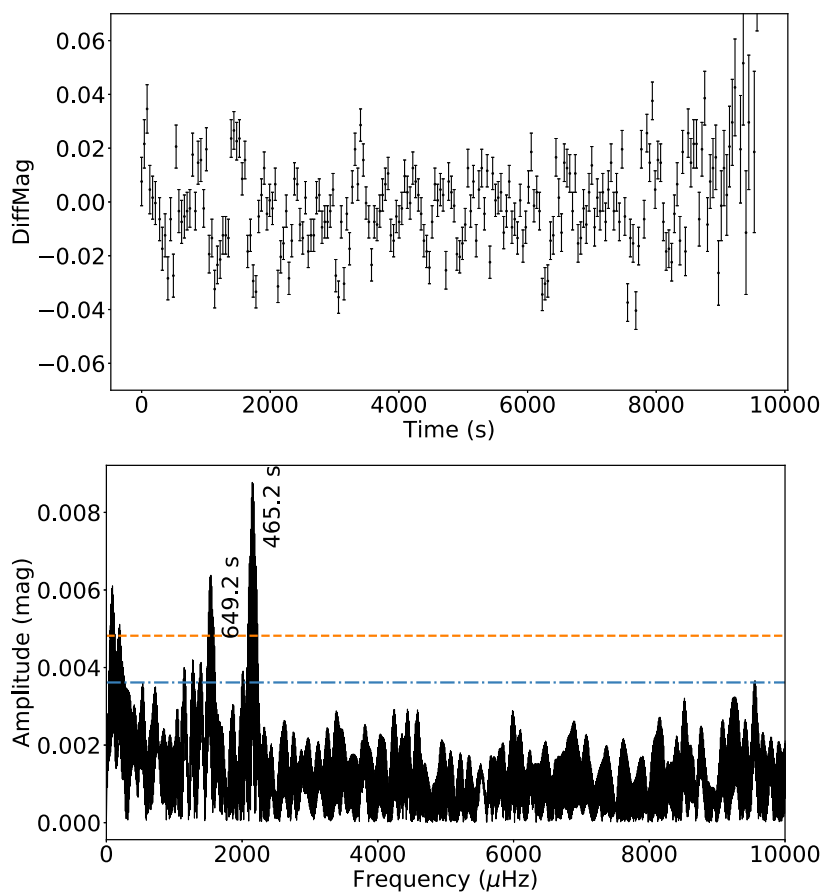

Figure 4. Light curve (top panel) and FT (bottom panel) for GD 195. The light curve corresponds to the $2.65 \mathrm{~h}$ run, while the FT corresponds to the sum of all observations. The orange dashed (blue dot-dashed) line corresponds to the $4 \sigma(3 \sigma)$ detection limit.
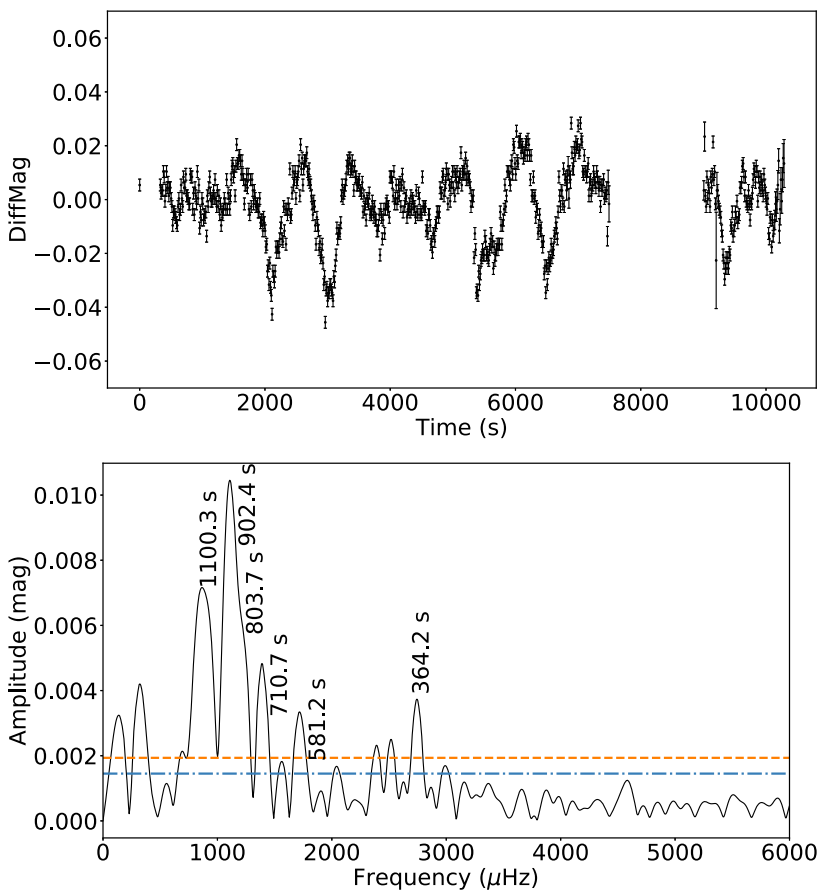

Figure 5. Light curve (top panel) and FT (bottom panel) for the star L495-82. The orange dashed (blue dot-dashed) line corresponds to the $4 \sigma(3 \sigma)$ detection limit. 
Table 3. Detected modes for the new pulsators. Column 1 lists the name, while the frequency, period, and amplitude are listed in columns 2, 3, and 4, respectively. Column 5 shows the identification of the mode, if it is a normal mode or a linear combination.

\begin{tabular}{lcccc}
\hline Star & $\begin{array}{c}\text { Freq } \\
(\mu \mathrm{Hz})\end{array}$ & $\begin{array}{c}\text { Period } \\
(\mathrm{s})\end{array}$ & $\begin{array}{c}\text { Amp } \\
(\mathrm{mma})\end{array}$ & $\mathrm{ID}$ \\
\hline SDSS J082804.63+094956.6 & 3499.073 & 285.79 & 14.0 & $\mathrm{f}_{1}$ \\
& 5093.984 & 196.31 & 10.9 & $\mathrm{f}_{2}$ \\
& 3909.610 & 255.78 & 5.0 & $\mathrm{f}_{3}$ \\
SDSS J094929.09+101918.8 & 5017.309 & 199.31 & 4.7 & $\mathrm{f}_{1}$ \\
& 3434.066 & 291.20 & 1.9 & $\mathrm{f}_{2}$ \\
& 8403.361 & 119.00 & 2.0 & $\mathrm{f}_{1}+\mathrm{f}_{2}$ \\
& 2149.798 & 465.16 & 8.7 & $\mathrm{f}_{1}$ \\
GD 195 & 1540.357 & 649.20 & 6.2 & $\mathrm{f}_{2}$ \\
& 1108.125 & 902.425 & 10.48 & $\mathrm{f}_{1}$ \\
& 908.856 & 1100.283 & 6.72 & $\mathrm{f}_{2}$ \\
& 1244.210 & 803.722 & 5.74 & $\mathrm{f}_{3}$ \\
& 1404.597 & 711.947 & 3.92 & $\mathrm{f}_{4}$ \\
& 2746.011 & 364.164 & 3.59 & $\mathrm{f}_{5}$ \\
& 991.480 & 1008.590 & 3.74 & $\mathrm{f}_{6}$ \\
& 1720.509 & 581.223 & 2.62 & $\mathrm{f}_{7}$ \\
& 2396.375 & 417.296 & 2.00 & $\mathrm{f}_{4}+\mathrm{f}_{6}$ \\
& 4578.305 & 218.421 & 1.41 & $f_{8}$ \\
2031.562 & 492.23 & 1.39 & $f_{9}$ \\
& 3018.182 & 331.32 & 1.21 & $\mathrm{f}_{6}+\mathrm{f}_{10}$ \\
& 2541.883 & 393.409 & 1.19 & $\mathrm{f}_{8}-\mathrm{f}_{9}$ \\
\hline & & & & \\
\hline
\end{tabular}

the dashed and dot-dashed lines correspond to the $3 \sigma$ and $4 \sigma$ limit, respectively. The FT shows three well-defined peaks above the $4 \sigma$ limit in the high-frequency domain. The detected frequencies and periods and the corresponding amplitudes are listed in Table 3. The modes show short periods between 196 and $285 \mathrm{~s}$, corresponding to a blue edge pulsator. From the FT we were not able to detect any multiplets, so no harmonic degree can be obtained directly from the observations.

\subsubsection{SDSS J094929.09+101918.8}

SDSS J094929.09+101918.8 was also selected from the SDSS catalogue presented by Kepler et al. (2016, 2019). From spectroscopy the star has a stellar mass of $0.644 \mathrm{M}_{\odot}$ and a 3D effective temperature of $11665 \mathrm{~K}$. It was observed for a total of $3.36 \mathrm{~h}$ on the SOAR telescope. In Fig. 3 we present the light curve for the $2.07 \mathrm{~h}$ observation run and the FT for all observations. As can be seen from the FT, this object shows one period of $199.31 \mathrm{~s}$ above the $4 \sigma$ limit. For amplitudes lower than $4 \sigma$, but higher than $3 \sigma$, we found two additional periods. In particular, the mode with a period of $291.20 \mathrm{~s}$ is present only in the second observation night, while the period of $119 \mathrm{~s}$ appears when we combine all observations, and it is a linear combination of the other two modes.

\subsubsection{GD 195}

GD 195 was classified as a very hot white dwarf star by Gianninas et al. (2011), with atmospheric parameters of $T_{\text {eff }}=14590 \pm 277 \mathrm{~K}$ and $\log g=7.82 \pm 0.05$. However, Kepler et al. (2016) found an effective temperature of $T_{\text {eff }}=11833 \pm 49 \mathrm{~K}$ and $\log g=$ $8.163 \pm 0.024$ based on SDSS spectra fitted with an updated version of the atmospheric models from Koester (2010) (see also Kepler et al. 2019). Additional fitting using a grid of updated models from Koester (2010) with $\alpha=0.8$ and 0.7 showed that the spectroscopic effective temperature of GD 195 is lower than $12000 \mathrm{~K}$, putting the star inside the classical ZZ Ceti instability strip (see Kepler et al. 2019, for details on the fitting procedure). Fig. 4 shows the FT corresponding to the combination of the three nights of observations. We found two modes with periods of 465.16 and $649.20 \mathrm{~s}$. According to the classification presented by Mukadam et al. (2006), a ZZ Ceti with period longer than $\sim 350 \mathrm{~s}$ corresponds to a variable in the middle of the instability strip with $T_{\text {eff }} \sim 11600 \mathrm{~K}$. This is in better agreement with the spectroscopic determinations of Kepler et al. (2016).

\subsubsection{L495-82}

L495-82 was selected from the list of objects presented by Bédard et al. (2017). This star is quite bright as compared to the other observed targets, with a $g$ magnitude of 13.76. Fig. 5 depicts the light curve and FT for L495-82, which shows a collection of longperiod modes, with a dominant period in $902.4 \mathrm{~s}$, compatible with a red edge pulsator (Kepler 1993). As can be seen from Table 3 there are several linear combinations which is also characteristic of a cool ZZ Ceti.

Since we observed this object for only approximately three hours, we cannot define all modes accurately, especially around the dominant peak corresponding to a period of $902 \mathrm{~s}$. In particular, the mode $\mathrm{f}_{3}$ is close to the linear combination $\left(\mathrm{f}_{1}+\mathrm{f}_{4}\right) / 2=1256.36 \mu \mathrm{Hz}$, which is within the uncertainties given that the peak for $f_{3}$ has a width of $30 \mu \mathrm{Hz}$. Thus, for asteroseismological purposes we will consider the $\mathrm{f}_{4}$ as a real mode and $\mathrm{f}_{3}$ as a linear combination.

\subsection{Known pulsators}

In this work we performed a follow-up of 10 known ZZ Ceti stars. For most of them, this is the first time follow-up observations are published since the discovery of their variable nature. The results from the observations are summarized in Table 4, where we list the frequencies, periods, and amplitudes obtained in this work, and the data reported in previous works (see last column of the table). The FT for the objects for which we found modes with new periods are shown in Fig. 6. In some cases, low-amplitude peaks appear in the FT after the pre-whitening process is done.

\subsection{Possible variables}

For the candidates LP375-51, SDSS J095703.09+080504.8, SDSS J212441.27-073234.9, and SDSS J213159.88+010856.3 (see Table 2 ), we detected variability over the $3 \sigma$ detection limit but below $4 \sigma$ on the FT. In these cases, the signal-to-noise ratio $(\mathrm{S} / \mathrm{N})$ was not sufficient to confirm variability and these objects are only classified as candidates. The FT for these objects are shown in Fig. 7. For LP375-51 the FT shows a peak at $1099.2 \mathrm{~s}$. This long period is compatible with the low spectroscopic effective temperature reported for this object. On the other hand, the FT for SDSS J095703.09+080504.8 shows two peaks with periods of 120.2 and 72.2 s, compatible with a blue edge pulsator. Similar to SDSS J095703.09+080504.8, SDSS J212441.27-073234.9 shows a spectroscopic effective temperature characteristic of a blue edge pulsator, and a short period of $108.5 \mathrm{~s}$ in the FT. Finally, SDSS J213159.88+010856.3 shows one period at $304.7 \mathrm{~s}$, compatible with a warm ZZ Ceti, in agreement with its spectroscopic effective temperature. The second mode with period of $90.1 \mathrm{~s}$ is probably 
Table 4. List of periods characteristic of the known variables observed in this work. We list the periods (in s) detected in this work and their amplitudes (in mma) in columns 2 and 3, while the periods detected in previous works and their amplitudes are listed in columns 4 and 5, along with the references in column 6 .

\begin{tabular}{|c|c|c|c|c|c|}
\hline Star & $\begin{array}{l}\text { This work } \\
\text { Period }\end{array}$ & Amp & $\begin{array}{l}\text { Known } \\
\text { Period }\end{array}$ & Amp & Ref. \\
\hline BPM 30551 & $\begin{array}{l}831.031 \\
775.235 \\
959.780 \\
460.060 \\
986.357 \\
649.348\end{array}$ & $\begin{array}{r}11.2 \\
11.3 \\
7.7 \\
5.7 \\
5.5 \\
5.5\end{array}$ & $\begin{array}{l}606.8 \\
744.7 \\
682.7 \\
840.2\end{array}$ & $\begin{aligned} & 11.5 \\
& 10.5 \\
\sim & 10 \\
\sim & 10\end{aligned}$ & Hesser, Lasker \& Neupert (1976) \\
\hline SDSS J092511.63+050932.6 & 1247.46 & 8.0 & $\begin{array}{l}1127.14 \\
1264.29 \\
1159.00 \\
1341.00\end{array}$ & $\begin{array}{l}3.17 \\
3.05 \\
2.7 \\
4.0\end{array}$ & $\begin{array}{l}\text { Castanheira et al. (2010) } \\
\text { Romero et al. (2013) }\end{array}$ \\
\hline HS $1249+0426$ & 294.91 & 14.5 & 288.9 & 7.6 & Voss et al. (2006) \\
\hline WD1345-0055 & 195.24 & 8.9 & $\begin{array}{l}195.2 \\
254.4\end{array}$ & $\begin{array}{l}5.5 \\
2.4\end{array}$ & Mukadam et al. (2004) \\
\hline HE $1429-037$ & 821.74 & 56.93 & $\begin{array}{r}450.1 \\
826.4 \\
969.0 \\
1084.9\end{array}$ & $\begin{array}{l}10.2 \\
18.3 \\
12.7 \\
16.3\end{array}$ & Silvotti et al. (2005) \\
\hline SDSS J161218.08+083028.1 & 115.122 & 5.14 & 115.17 & 5.06 & Castanheira et al. (2013) \\
\hline GD 385 & $\begin{array}{l}256.09 \\
127.93\end{array}$ & $\begin{array}{l}9.4 \\
3.5\end{array}$ & $\begin{array}{l}256.12 \\
128.15\end{array}$ & $\begin{array}{r}11.4 \\
3.7\end{array}$ & Castanheira \& Kepler (2009) \\
\hline SDSS J215905.53+132255.8 & $\begin{array}{l}678.78 \\
746.67\end{array}$ & $\begin{array}{r}8.0 \\
24.2\end{array}$ & $\begin{array}{l}683.7 \\
801.0\end{array}$ & $\begin{array}{l}11.7 \\
15.1\end{array}$ & Mullally et al. (2005) \\
\hline SDSS J221458.37-002511.9 & 255.08 & 16.0 & $\begin{array}{l}255.2 \\
195.2\end{array}$ & $\begin{array}{r}13.1 \\
6.1\end{array}$ & Mullally et al. (2005) \\
\hline SDSS J235040.72-005430.9 & $\begin{array}{l}304.74 \\
390.32 \\
271.87\end{array}$ & $\begin{array}{c}18.29 \\
10.17 \\
8.2\end{array}$ & $\begin{array}{l}304.3 \\
391.1 \\
273.3 \\
206.7\end{array}$ & $\begin{array}{r}17.0 \\
7.5 \\
6.2 \\
3.2\end{array}$ & $\begin{array}{l}\text { Mukadam et al. (2004) } \\
\text { Mukadam et al. (2006) }\end{array}$ \\
\hline
\end{tabular}

instrumental due to the integration time of $30 \mathrm{~s}$. We list the periods between 3 and $4 \sigma$ in Table 5 . Further observations are required to confirm the variable nature of these stars.

\subsection{NOV}

From the observed sample, we did not detect any variability on the FT for four objects, within the detection limit given by the $\mathrm{S} / \mathrm{N}$, and thus they are classified as Not Observed to Vary (NOV). We list the objects in Table 6 along with the detection limit from our observations. We recommend a follow-up observations given that the detection limit is higher than the typical amplitudes observed in ZZ Cetis, especially near the blue edge of the instability strip (e.g. Castanheira et al. 2013).

\section{ASTEROSEISMOLOGICAL FITS}

In this section we present a detailed asteroseismological analysis of all observed objetcts, which showed variability. That includes the 10 known $\mathrm{ZZ}$ Cetis and the four new variables reported in this work. The DA white dwarf models used in this work are the result of full evolutionary computations of the progenitor stars, from the ZAMS, through the hydrogen and helium central burning stages, thermally pulsating and mass-loss AGB phase and finally the planetary nebulae domain. They were generated using the LPCODE evolutionary code (see Althaus et al. 2010; Renedo et al. 2010; Romero, Campos \& Kepler 2015, for details). The stellar mass values go from 0.493 to $1.05 \mathrm{M}_{\odot}$, with a hydrogen layer mass in the range of $\sim 4 \times 10^{-4}$ to $\sim 10^{-10} M_{*}$ depending on the stellar mass. Non-radial adiabatic $g$-mode pulsations were computed using the adiabatic version of the LP-PUL pulsation code described in Córsico \& Althaus (2006). We employ an extended version of the model grid presented in Romero et al. (2017) that includes six new cooling sequences with stellar masses between 0.5 and $0.7 \mathrm{M}_{\odot}$, along with approximately eight hydrogen layer values for each sequence, depending on the mass.

For each object we search for an asteroseismological representative model that best matches the observed periods. To this end, we seek for the theoretical model that minimizes the quality function given by Castanheira \& Kepler (2008):

$S\left(M_{*}, M_{\mathrm{H}}, T_{\mathrm{eff}}\right)=\sqrt{\sum_{i=1}^{N} \min \left[\frac{\left[\Pi_{k}^{\mathrm{th}}-\Pi_{k}^{\mathrm{obs}}\right]^{2} A_{i}}{\sum_{i=1}^{N} A_{i}}\right]}$,

where $\Pi^{\text {th }}$ is the theoretical period that better fits the observed $\Pi^{\text {obs }}$, and the amplitudes $A_{i}$ are used as weights for each period. In this 

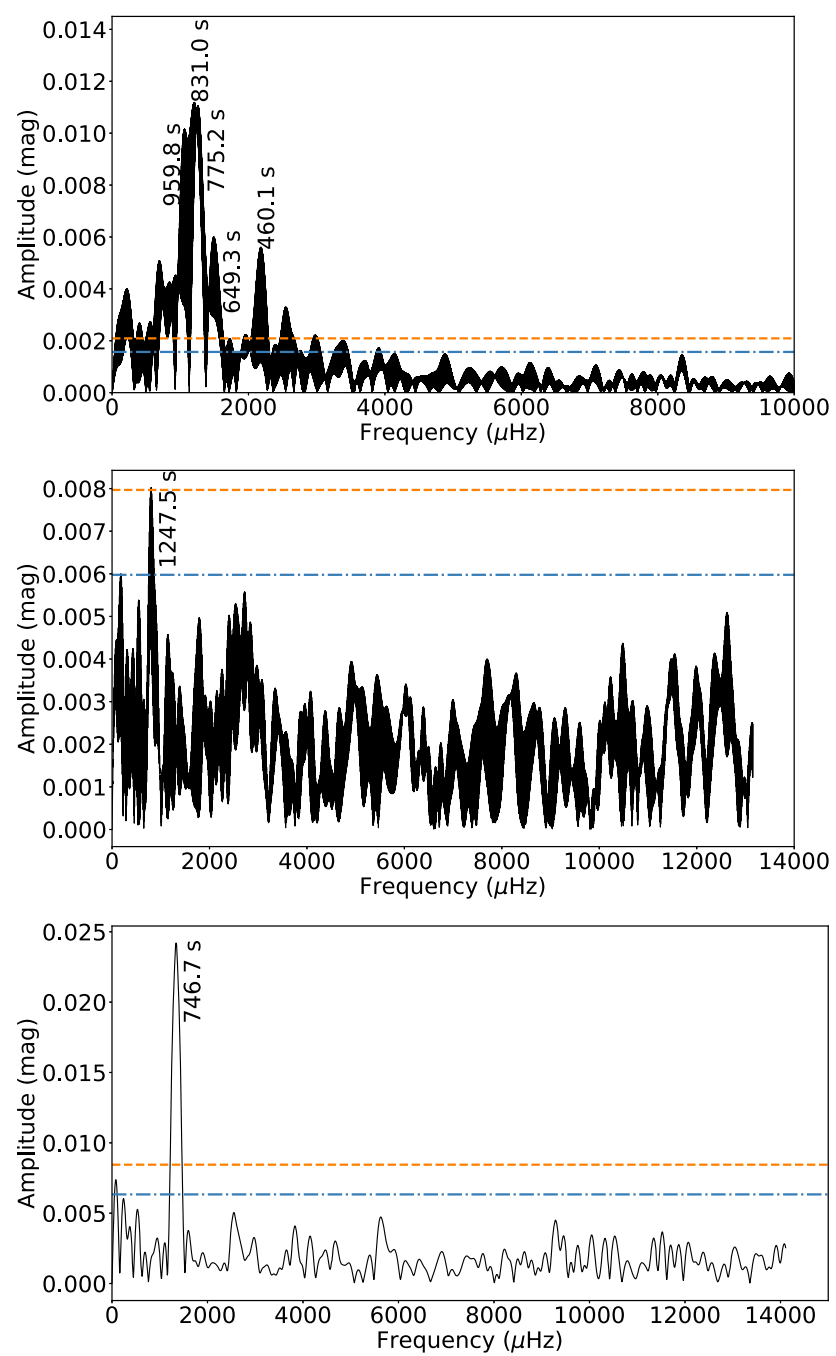

Figure 6. FT for the three known ZZ Cetis with new detected periods. From top to bottom: BPM 30551, SDSS J092511.63+050932.6, and SDSS J215905.53+132255.8. Note that the FT shows peaks above the $3 \sigma$ but below the $4 \sigma$ detection limit, adopted in this work.

way, the period fit is more influenced by those modes with large observed amplitudes.

The results of the asteroseismological fits are presented in Tables 7 and 8 , corresponding to the new variables and the known variables, respectively. For each object we list the effective temperature, stellar mass, and thickness of the hydrogen envelope for the seismological model, in columns 2, 3, and 4, respectively. Column 5 shows the value of the observed period while the theoretical periods are listed in column 6 along with the harmonic degree ( $\mathrm{col} 7)$ and the radial order (col 8). Finally, the value of the quality function $S$ is listed in column 9. The first model listed is the one we choose to be the best-fitting model for that particular object.

In Table 9 we list the structural parameters of the asteroseismological models selected as best-fitting models for each star analysed in this paper. The uncertainties for $M_{*}, T_{\text {eff }}$, and $\log \left(L / L_{\odot}\right)$ were computed using the following expression (Zhang, Robinson \& Nather 1986; Castanheira \& Kepler 2008):

$\sigma_{i}^{2}=\frac{d_{i}^{2}}{\left(S-S_{0}\right)}$,
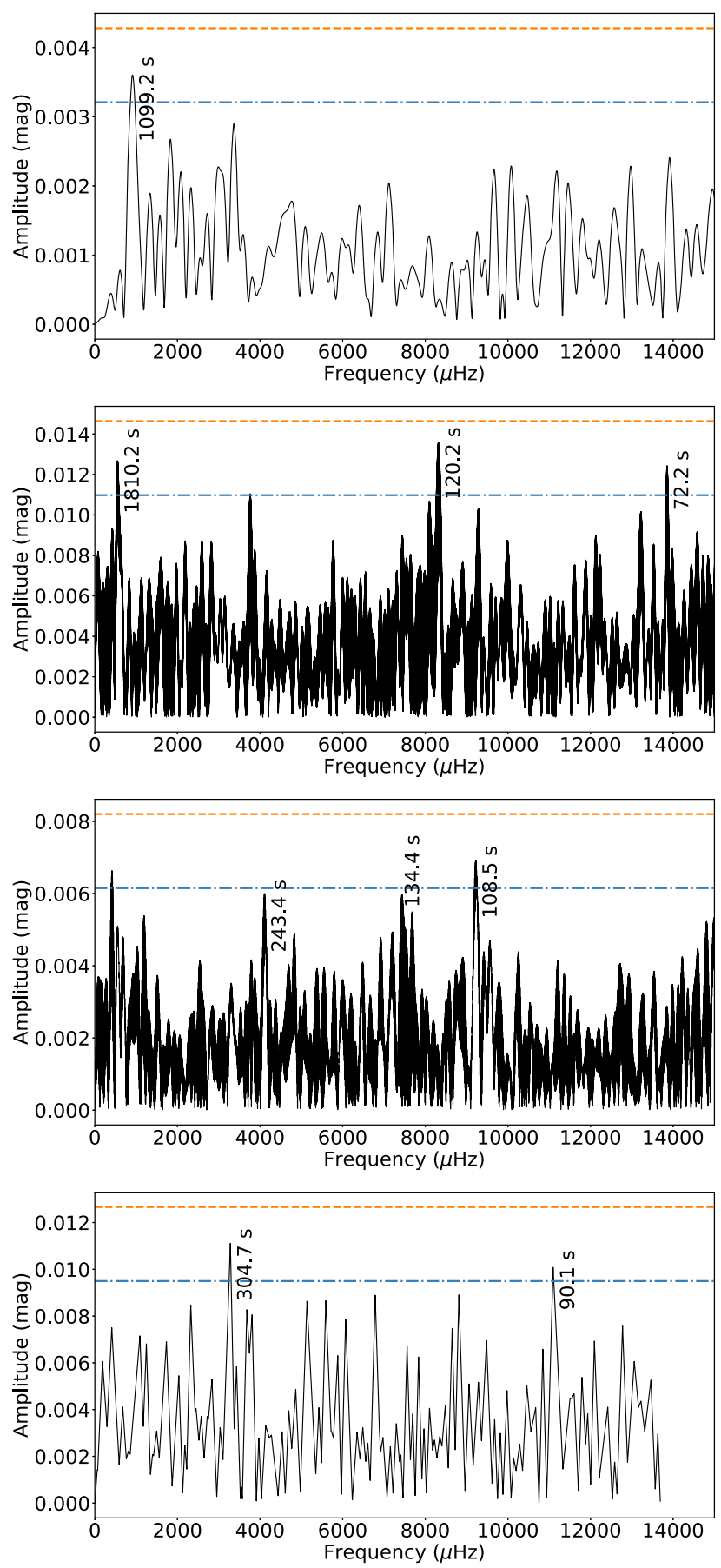

Figure 7. FT for the four objects classified as possible variables From top to bottom: LP375-51, SDSS J095703.09+080504.8, SDSS J212441.27-073234.9, and SDSS J213159.88+010856.3. Note that the FT shows peaks above the $3 \sigma$ but below the $4 \sigma$ detection limit, adopted in this work.

where $S_{0}=S\left(M_{*}^{0}, M_{\mathrm{H}}^{0}, T_{\mathrm{eff}}^{0}\right)$ is the minimum of the quality function $S$ reached at $\left(M_{*}^{0}, M_{\mathrm{H}}^{0}, T_{\mathrm{eff}}^{0}\right)$, and $S$ is the value of the quality function when we change the parameter $i$ by an amount $d_{i}$, keeping fixed the other parameters. The uncertainties in the remaining quantities are derived from the uncertainties in $M_{*}, T_{\text {eff }}$, and $\log \left(L / L_{\odot}\right)$. These uncertainties represent the internal errors of the fitting procedure. 
Table 5. Possible variables found in this work, showing peaks between 3 and $4 \sigma$ in the FT. We include the frequency, period, and amplitude for each peak, in columns 2,3 , and 4 , respectively. In column 5 we list the $4 \sigma$ limit (mma). *Peak with amplitude below $3 \sigma$.

\begin{tabular}{lcccc}
\hline Star & $\begin{array}{c}\text { Freq } \\
(\mu \mathrm{Hz})\end{array}$ & $\begin{array}{c}\text { Period } \\
(\mathrm{s})\end{array}$ & $\begin{array}{c}\text { Amp } \\
(\mathrm{mma})\end{array}$ & $4 \sigma$ \\
\hline SDSS J095703.09+080504.8 & 8321.95 & 120.2 & 12.9 & 14.63 \\
& 552.48 & 1810.02 & 13.5 & \\
& 13850.6 & 72.2 & 11.7 & \\
LP 375-51 & 909.75 & 1099.2 & 3.6 & 4.28 \\
SDSS J212441.27-073234.9 & 9218.58 & 108.5 & 6.9 & 8.20 \\
& 4108.62 & 243.4 & $5.9^{*}$ & \\
SDSS J213159.88+010856.3 & 3438.69 & 134.4 & $5.9^{*}$ & \\
\hline
\end{tabular}

Table 6. Objects with no detected periodicities. We include the magnitude in the $g$ filter (column 2) and the amplitude of the noise in the FT, as a detection limit (column 3).

\begin{tabular}{lcc}
\hline Star & $\mathrm{g}$ & $4 \sigma(\mathrm{mma})$ \\
\hline SDSS J113325.69+183934.7 & 17.59 & 8 \\
WD 1454-0111 & 17.34 & 10 \\
SDSS J161005.17+032356.1 & 18.55 & 7 \\
SDSS J235932.80-033541.1 & 17.91 & 2 \\
\hline
\end{tabular}

\subsection{New ZZ Cetis}

In this section we describe in detail the asteroseismological fits for the four new ZZ Ceti stars discovered in this paper. The results are presented in Table 7 .

\subsubsection{SDSS J082804.63+094956.6}

The new ZZ Ceti SDSS J082804+094956.6 shows three periods, with the mode at $285.79 \mathrm{~s}$ having the largest amplitude. This star shows period pulsations, shorter than $350 \mathrm{~s}$, so we expect it to be close to the blue edge of the instability strip. However, the 3Dcorrected spectroscopic effective temperature of $11691 \pm 53 \mathrm{~K}$ is closer to the middle of instability strip for the stellar mass of SDSS J082804.63+094956.6. The results from the seismological fit are listed in Table 7. The first model corresponds to a fit with all modes with $\ell=1$, while for the second we allowed the mode with $255.78 \mathrm{~s}$ to be either $\ell=1$ or 2 . Both models are characterized by an effective temperature around $11600 \mathrm{~K}$, in agreement with the spectroscopic value. The hydrogen envelope is thinner than the canonical value, but is still considered a thick envelope.

\subsubsection{SDSS J094929.09+101918.8}

This star shows one period above the $4 \sigma$ detection limit, with a period of $199.31 \mathrm{~s}$, thus we consider this period for our seismological fit. Since we only have one period we need to make some additional restrictions to obtain a theoretical representative model. From spectroscopy, Kepler et al. (2019) obtained a 3D-corrected effective temperature and surface gravity of $11685 \pm 65 \mathrm{~K}$ and $\log g=$ $8.073 \pm 0.034$, leading to a stellar mass of $0.664 \pm 0.027 \mathrm{M}_{\odot}$. The photometric temperature obtained from the SDSS filters (see Ourique et al. 2019, for details on the procedure) is $11700 \pm 187 \mathrm{~K}$ with $\log g=8.00 \pm 0.1$ in agreement with the spectroscopy from Kepler et al. (2019).

For our seismological fit we consider only the mode with a period of $\sim 199 \mathrm{~s}$, which is the only one with an amplitude larger than $4 \sigma$ in the FT. The results from our seismological fit are listed in Table 7. The solution is characterized by a stellar mass of $0.705 \mathrm{M}_{\odot}$ and a thick hydrogen envelope. The best-fitting model for SDSS J094929.09+101918.8 has a period of $292.31 \mathrm{~s}$, with $\ell=2$ and $k=$ 9 that can fit the mode with a period $291.20 \mathrm{~s}$.

\subsubsection{GD 195}

From our observations we find two pulsation modes for GD 195, with periods of 465 and 649 s. For these period values we expect the star to be a warm ZZ Ceti, with effective temperature around $\sim 11500 \mathrm{~K}$, located in the middle of the instability strip. Since the modes show similar amplitudes in the FT, we consider that both have the same harmonic degree. In this case we expect a degeneracy in the solutions, and we need to use an additional restriction, which in this case can be the spectroscopic temperature and mass. The seismic solution compatible with the spectroscopic determinations is characterized by an effective temperature near the blue edge of the instability strip. The solution also shows a thick hydrogen envelope, considering that the stellar mass is $0.705 \mathrm{M}_{\odot}$ (see Table 7 for details). A second solution, with a lower value of the quality function, is found when we relax the condition on the effective temperature. The stellar mass is somewhat larger but the effective temperature is $\sim 11000 \mathrm{~K}$, closer to the red edge of the instability strip. Also, the hydrogen envelope mass is the thinnest of our model grid for this stellar mass. A lower effective temperature is compatible with the observed periods, being larger than $\sim 350 \mathrm{~s}$ (Mukadam et al. 2006). In addition, a low effective temperature is compatible with the colours from Gaia for this object leading to an effective temperature of $\sim 11000 \mathrm{~K}$ (see Section 6).

\section{1 .4 L495-82}

L495-82 is a rich pulsator with seven detected modes. This is compatible with its low effective temperature of $11029 \pm 160 \mathrm{~K}$. We consider seven periods in our seismological fit, as shown in Table 7. As an additional restriction, we consider the mode with the largest amplitude, and a period of $902.42 \mathrm{~s}$, to be $\ell=1$. We obtain the bestfitting model with a stellar mass of $0.593 \mathrm{M}_{\odot}$ and a low effective temperature, compatible with the values from spectroscopic and Gaia colours (see Section 6). The hydrogen envelope corresponds to a thick envelope. Since the star shows a period of $365.16 \mathrm{~s}$, we consider it to be too short for a pulsator near the red edge of the instability strip, and more characteristic of warm ZZ Ceti, with an effective temperature of $\sim 11500 \mathrm{~K}$ (Mukadam et al. 2006). With this consideration, we found a second minimum of the quality function characterized by an effective temperature of $\sim 11600 \mathrm{~K}$. However, the hydrogen envelope is a factor of 100 thinner than the previous model.

\subsection{Known variables}

We present the asteroseismological fits for the known ZZ Ceti stars that were observed in this work. For the fit we consider all the periods observed for each object, listed in the columns 2 and 4 of Table 4. When a detected frequency is close to one previously detected by other authors, we consider the uncertainties in the frequency to 
Table 7. Best-fitting model for the four new ZZ Ceti stars. The effective temperature, stellar mass, and the mass of the hydrogen envelope are listed in columns 2,3 , and 4 , respectively. We list the observed periods used in the asteroseismological fit in column 5 . The theoretical periods, harmonic degree, and radial order are listed in columns 6, 7, and 8, respectively. The value of the quality function $S$ in seconds is listed in column 9 .

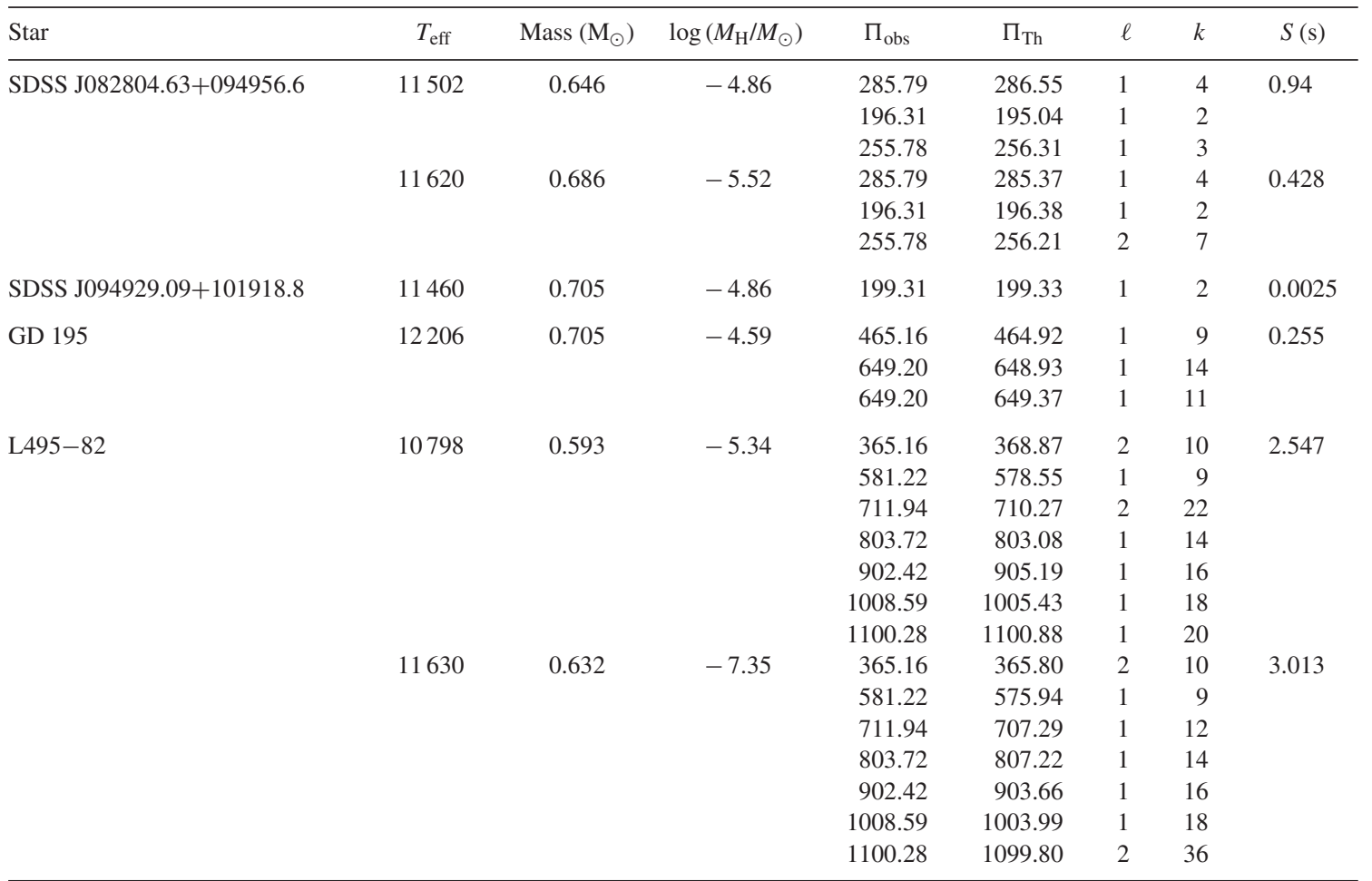

determine whether it is a new mode or not. The results of our seismological fit for the known ZZ Cetis are listed in Table 8. We present the fitting process for each object below.

BPM 30551: BPM 30551 was observed by Hesser et al. (1976). Several periods were detected between $\sim 300$ and $\sim 2300 \mathrm{~s}$ in the 10 nights. In previous seismological studies, only two periods were used, with 606.8 and $744.7 \mathrm{~s}$ (Castanheira \& Kepler 2009; Romero et al. 2013, 2012). For our seismological fit we consider the six modes detected in this work, with periods between 460 and $986 \mathrm{~s}$. As a result we find the best-fitting model characterized by a stellar mass of $0.632 \mathrm{M}_{\odot}$ and a thin envelope with $\sim 5 \times 10^{-9} M_{*}$.

SDSS J092511.63+050932.6: This star is one of the coolest ZZ Cetis, with a spectroscopic effective temperature less than $\sim 11000 \mathrm{~K}$. From the FT we detected one period of $1247.5 \mathrm{~s}$. Considering the uncertainty in the frequency for this period of $198 \mu \mathrm{Hz}$, we consider it to be the same mode as the one with a period of $1264.3 \mathrm{~s}$, detected by Castanheira et al. (2010), with a difference of $\delta v=10.6 \mu \mathrm{Hz}$ between both determinations. For our seismological fit, we consider the mean frequency, corresponding to a period of $1255.84 \mathrm{~s}$, along with the other three periods detected in previous works. If we fix the harmonic degree to be $\ell=1$ for all modes we obtain a representative model with $0.675 \mathrm{M}_{\odot}$ and a thick hydrogen envelope. By relaxing this condition, two periods are fitted with quadrupole $(\ell=2)$ mode. The solution has a larger mass and a thinner hydrogen envelope, possibly due to the core-envelope symmetry (Montgomery et al. 2003).

HS 1249+0426: This object shows only one peak in the FT at $294.91 \mathrm{~s}$. Voss et al. (2006) also detected one period of $288.9 \mathrm{~s}$. Given the uncertainty in the frequency, we concluded that they are the same period, and use the value obtained in this work for the seismological fit. We consider only $\ell=1$ modes in our fit. The solution is similar to that found by Romero et al. (2012), characterized by a canonical stellar mass and an effective temperature of $\sim 11500 \mathrm{~K}$. Finally, the hydrogen envelope is a factor of three thinner than the previous fit, but still considered a thick envelope.

WD1345-0055: Mukadam et al. (2004) reported the detection of two short periods for WD1345-0055. From our observations we recover the one period of $195.2 \mathrm{~s}$. For our seismological fit, we consider the two modes. As a result we found a representative model with a stellar mass of $0.686 \mathrm{M}_{\odot}$ and a canonical envelope, that predicted by single stellar evolution for this stellar mass. Both modes are fitted with theoretical dipole $(\ell=1)$ mode.

HE 1429-037: For this object, Silvotti et al. (2005) reported the detection of four periods between 450 and 1084 s. From our observations we found one mode with a period of $821.74 \mathrm{~s}$. Considering the uncertainties we conclude that this period corresponds to the period of $829.3 \mathrm{~s}$, detected by Silvotti et al. (2005). For the seismological fit we consider the mean frequency, corresponding to a period of $825.505 \mathrm{~s}$. The seismic solution has a low stellar mass of $0.548 \mathrm{M}_{\odot}$ and a thick hydrogen envelope of $1.9 \times 10^{-5} M_{*}$.

SDSS J161218.08+083028.1: Castanheira et al. (2013) reported the detection of two short periods, part of a triplet with a central component with a period of $\sim 115 \mathrm{~s}$. We recover these periods from our observations, with the additional possible detection of a period of $112.09 \mathrm{~s}$, which is part of the triplet. We consider the spectroscopic determinations of mass and $T_{\text {eff }}$ as additional restrictions in our seismological fit, since we only have one observed mode. The seismic solution has a high stellar mass and effective temperature, as expected from a short-period pulsator, with a 
Table 8. Best-fitting model for the known ZZ Cetis, using the list of observed modes (see the text for details). The effective temperature, stellar mass, and the mass of the hydrogen envelope are listed in columns 2, 3, and 4, respectively. We list the observed periods used in the asteroseismological fit in column 5. The theoretical periods, harmonic degree, and radial order are listed in columns 6, 7, and 8, respectively. The value of the quality function $S$ in seconds is listed in column 9.

\begin{tabular}{|c|c|c|c|c|c|c|c|c|}
\hline Star & $T_{\text {eff }}$ & Mass $\left(\mathrm{M}_{\odot}\right)$ & $\log \left(M_{\mathrm{H}} / M_{\odot}\right)$ & $\Pi_{\mathrm{obs}}$ & $\Pi_{\mathrm{Th}}$ & $\ell$ & $k$ & $S(\mathrm{~s})$ \\
\hline BPM 30551 & 11578 & 0.632 & -8.33 & $\begin{array}{l}460.06 \\
649.35 \\
775.23 \\
831.03 \\
959.78 \\
986.36\end{array}$ & $\begin{array}{l}459.97 \\
647.17 \\
772.91 \\
832.45 \\
958.68 \\
987.60\end{array}$ & $\begin{array}{l}2 \\
1 \\
1 \\
1 \\
2 \\
2\end{array}$ & $\begin{array}{l}13 \\
10 \\
13 \\
14 \\
30 \\
31\end{array}$ & 1.86 \\
\hline SDSS J092511.63+050932.6 & 11385 & 0.675 & -4.87 & $\begin{array}{l}1127.10 \\
1159.00 \\
1255.84 \\
1341.00\end{array}$ & $\begin{array}{l}1127.01 \\
1163.08 \\
1255.74 \\
1339.61\end{array}$ & $\begin{array}{l}1 \\
1 \\
1 \\
1\end{array}$ & $\begin{array}{l}24 \\
25 \\
27 \\
29\end{array}$ & 1.89 \\
\hline & 11241 & 0.705 & -7.35 & $\begin{array}{l}1127.10 \\
1159.00 \\
1255.84 \\
1341.00\end{array}$ & $\begin{array}{l}1127.93 \\
1159.12 \\
1255.09 \\
1341.49\end{array}$ & $\begin{array}{l}2 \\
2 \\
1 \\
1\end{array}$ & $\begin{array}{l}38 \\
39 \\
24 \\
26\end{array}$ & 0.64 \\
\hline HS $1249+0426$ & 11564 & 0.609 & -4.85 & 294.89 & 294.90 & 1 & 4 & 0.001 \\
\hline WD1345-0655 & 11676 & 0.686 & -4.36 & $\begin{array}{l}195.2 \\
254.4\end{array}$ & $\begin{array}{l}194.94 \\
254.47\end{array}$ & $\begin{array}{l}1 \\
1\end{array}$ & $\begin{array}{l}2 \\
3\end{array}$ & 0.22 \\
\hline HE 1429-037 & 11404 & 0.548 & -4.27 & $\begin{array}{c}450.10 \\
821.74 \\
969.00 \\
1084.90\end{array}$ & $\begin{array}{c}452.39 \\
821.03 \\
969.70 \\
1083.92\end{array}$ & $\begin{array}{l}1 \\
1 \\
2 \\
1\end{array}$ & $\begin{array}{c}7 \\
15 \\
33 \\
21\end{array}$ & 1.29 \\
\hline SDSS J161218.08+083028.1 & $\begin{array}{l}12312 \\
12619\end{array}$ & $\begin{array}{l}0.878 \\
0.686\end{array}$ & $\begin{array}{l}-5.54 \\
-4.36\end{array}$ & $\begin{array}{l}115.122 \\
115.122\end{array}$ & $\begin{array}{l}115.187 \\
115.123\end{array}$ & $\begin{array}{l}1 \\
1\end{array}$ & $\begin{array}{l}1 \\
1\end{array}$ & $\begin{array}{l}0.033 \\
0.001\end{array}$ \\
\hline GD 385 & $\begin{array}{l}12147 \\
11560\end{array}$ & $\begin{array}{l}0.800 \\
0.646\end{array}$ & $\begin{array}{l}-5.39 \\
-6.34\end{array}$ & $\begin{array}{l}127.93 \\
256.09 \\
127.93 \\
256.09\end{array}$ & $\begin{array}{l}127.53 \\
256.14 \\
127.79 \\
256.31\end{array}$ & $\begin{array}{l}1 \\
1 \\
2 \\
1\end{array}$ & $\begin{array}{l}1 \\
4 \\
2 \\
3\end{array}$ & 0.22 \\
\hline SDSS J215905.53+132255.8 & 11771 & 0.917 & -5.41 & $\begin{array}{l}683.70 \\
746.67 \\
801.00\end{array}$ & $\begin{array}{l}684.39 \\
746.32 \\
800.97\end{array}$ & $\begin{array}{l}1 \\
2 \\
2\end{array}$ & $\begin{array}{l}18 \\
35 \\
38\end{array}$ & 0.58 \\
\hline & 11688 & 0.976 & -6.46 & $\begin{array}{l}683.70 \\
746.67 \\
801.00\end{array}$ & $\begin{array}{l}684.71 \\
746.19 \\
801.51\end{array}$ & $\begin{array}{l}1 \\
2 \\
2\end{array}$ & $\begin{array}{l}17 \\
33 \\
36\end{array}$ & 1.09 \\
\hline SDSS J221458.37-002511.9 & 11568 & 0.878 & -7.38 & $\begin{array}{l}195.08 \\
255.20\end{array}$ & $\begin{array}{l}195.61 \\
255.10\end{array}$ & $\begin{array}{l}1 \\
1\end{array}$ & $\begin{array}{l}2 \\
4\end{array}$ & 0.22 \\
\hline & 11605 & 0.686 & -4.36 & $\begin{array}{l}195.08 \\
255.20\end{array}$ & $\begin{array}{l}195.50 \\
254.87\end{array}$ & $\begin{array}{l}1 \\
1\end{array}$ & $\begin{array}{l}2 \\
4\end{array}$ & 0.24 \\
\hline SDSS J235040.72-005430.9 & 10061 & 0.690 & -7.35 & $\begin{array}{l}271.87 \\
304.74 \\
390.32\end{array}$ & $\begin{array}{l}272.93 \\
303.67 \\
391.08\end{array}$ & $\begin{array}{l}1 \\
1 \\
1\end{array}$ & $\begin{array}{l}3 \\
4 \\
5\end{array}$ & 0.99 \\
\hline & 10290 & 0.660 & -7.33 & $\begin{array}{l}271.87 \\
304.74 \\
390.32\end{array}$ & $\begin{array}{l}272.12 \\
304.55 \\
389.93\end{array}$ & $\begin{array}{l}2 \\
1 \\
1\end{array}$ & $\begin{array}{l}6 \\
4 \\
5\end{array}$ & 0.31 \\
\hline
\end{tabular}

hydrogen envelope of $2.85 \times 10^{-6} M_{*}$. If we relax the restriction in stellar mass, we found a second solution with a $0.686 \mathrm{M}_{\odot}$ and a thicker envelope.

GD 385: GD 385 is a hot ZZ Ceti showing two modes. We recover both modes from our observations and did not detected new periodicities. For our seismological fit, first we fixed the harmonic degree to $\ell=1$ for both modes and obtained a hot solution with a stellar mass of $0.8 \mathrm{M}_{\odot}$, somewhat larger than the spectroscopic mass (see Table 1). The second solution presented in Table 8 was obtained by fixing the mode with the largest amplitude to be a dipole mode and letting the harmonic degree for the second mode free. The solution shows a stellar mass compatible with the spectroscopy but the effective temperature is low, as compared to other pulsators that show a period $\sim 195 \mathrm{~s}$.

SDSS J215905.53+132255.8: This object is the most massive ZZ Ceti analysed in this work. Two pulsation modes were reported by Mullally et al. (2005), with periods of 683.7 and $801.0 \mathrm{~s}$. In this work we find a period of $746.67 \mathrm{~s}$ with a large amplitude, and a second period with $678.8 \mathrm{~s}$ after subtracting the main peak from the FT. The second period has a frequency that is $\delta v=10 \mu \mathrm{Hz}$ from 
Table 9. Structural parameters for the best-fitting models corresponding to each DAV star analysed in this paper. The uncertainties are the internal errors of the fitting procedure.

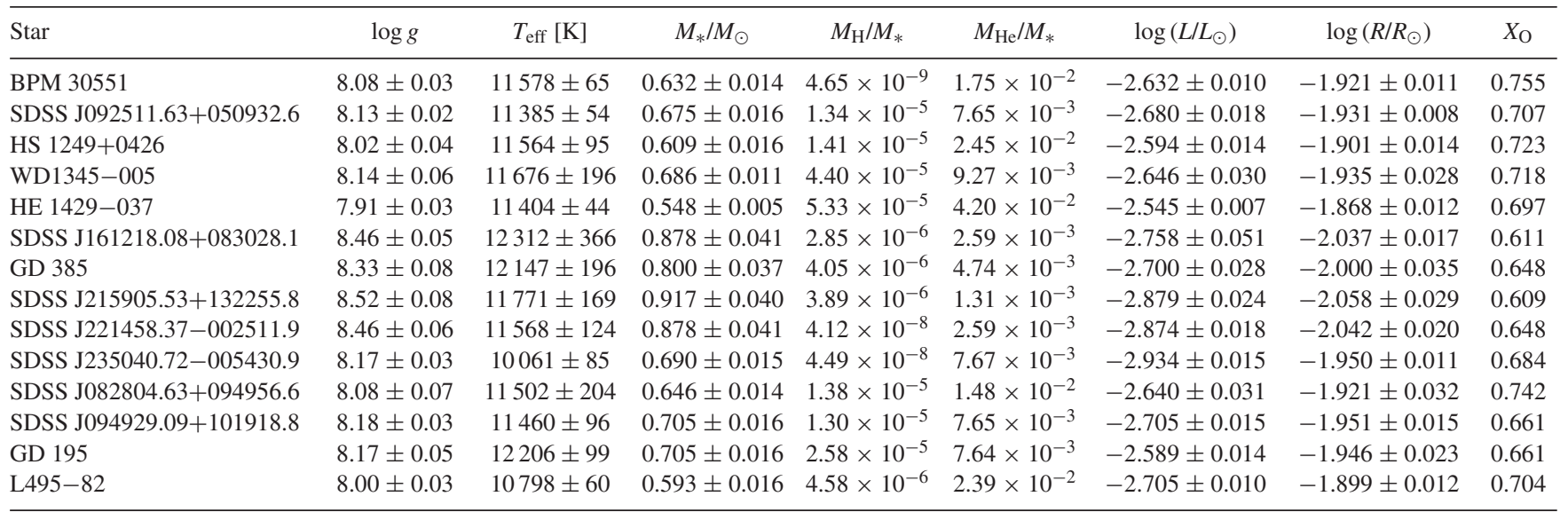

the frequency corresponding to the mode with $683.7 \mathrm{~s}$ previously reported. Thus we consider that they are the same mode and use three periods in our seismological fit. The model that minimized the quality function is characterized by a stellar mass of $0.917 \mathrm{M}_{\odot}$ as it is shown in Table 8. We also consider a second solution, closer to the one obtained by Romero et al. (2013) using two periods. In this case, the stellar mass is $0.976 \mathrm{M}_{\odot}$ and the hydrogen envelope is $\sim 10$ times thinner than the first solution, which is related to the core-envelope symmetry (Montgomery et al. 2003). In this case the core should be 7 per cent crystallized.

SDSS J221458.37-002511.9: For this object, we recover one of the two periods presented by Mullally et al. (2005), with a period of $\sim 255 \mathrm{~s}$. For our seismological fit we use the two known periods. We find two representative theoretical models with similar quality functions, listed in Table 8 . The first model has a stellar mass of $0.878 \mathrm{M}_{\odot}$ and a thin hydrogen envelope, while the second solution is characterized by a stellar mass of $0.686 \mathrm{M}_{\odot}$ and a thick envelope. Both models fit the observed modes with $\ell=1$ modes and show effective temperatures of $\sim 11600 \mathrm{~K}$, in agreement with the spectroscopy.

SDSS J235040.72-005430.9: This ZZ Ceti is an ultra-cool ZZ Ceti, with an spectroscopic effective temperature of $\sim 10600 \mathrm{~K}$. From our observations we recover three modes, presented in Mukadam et al. (2004). We carried two seismological fits, one fixing the harmonic degree to be $\ell=1$ for all modes, and a second by considering that the mode with the highest amplitude is a $\ell=1$ mode while leaving the harmonic degree free for the remaining two modes. Both fitting procedures lead to a cool solution with a thin hydrogen envelope $\log \left(M_{\mathrm{H}} / M_{*}\right) \sim-7.3$.

This object is very odd in the sense that the effective temperature is very low as compared with the bulk of ZZ Ceti stars. Romero et al. (2013) considered that this object, and other ultra-cool ZZ Cetis, could be low-mass white dwarfs, with stellar masses below $0.3 \mathrm{M}_{\odot}$, which is in line with the mass obtained from parallax (see Section 6). Other explanation include the possibility of a binary companion, in which case, the determination of the spectroscopic mass being affected by the presence of the companion (Fuchs 2018). This hypothesis will be explored in a future paper.

To summarize, in Fig. 8 we plot all the seismological solutions listed in Tables 7 and 8, in the stellar mass-thickness of the hydrogen envelope plane. With black circles, we plot the bestfitting models for each star, whereas the blue squares represent the second solutions, when present. Solutions corresponding to

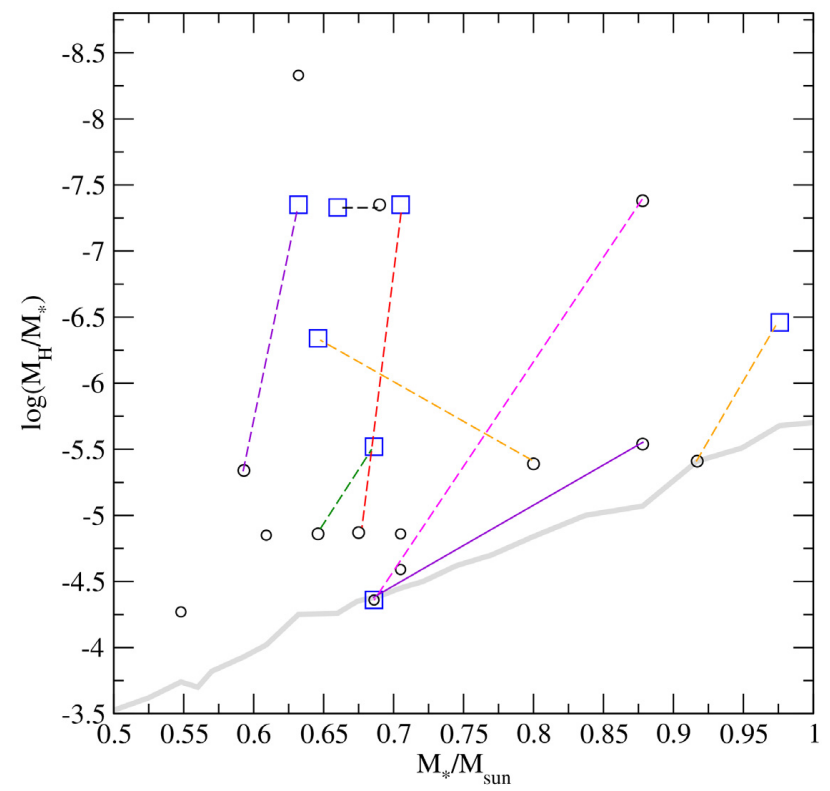

Figure 8. Values of hydrogen envelope mass in terms of the stellar mass, corresponding to all the asteroseismological models of the 14 objects analysed in this work. The black circles and blue squares correspond to the first and second solution, respectively (see Tables 7 and 8). Solutions corresponding to the same object are connected with a line. The thick grey line depicts the canonical values of the hydrogen envelope thickness (Romero et al. 2017).

the same object are joint together with a line. The thick grey line indicates the high limit of the hydrogen mass, as predicted by stellar evolution. Note that for several objects, we obtain two possible seismological solutions, even after additional restrictions are considered. Usually one is characterized by a higher stellar mass and a thin hydrogen envelope and other characterized by a lower mass and a thicker hydrogen layer. For example, for L495-82 we obtained the best-fitting model characterized by $M_{*}=0.593 \mathrm{M}_{\odot}$ and $\log \left(M_{\mathrm{H}} / M_{*}\right)=-5.34$ and a second solution with $M_{*}=0.632 \mathrm{M}_{\odot}$ and $\log \left(M_{\mathrm{H}} / M_{*}\right)=-7.35$. This degeneracy in solutions is related to the so-called 'core-envelope symmetry' discussed in Montgomery et al. (2003), where a sharp feature in the Brunt-Väisälä frequency in the envelope can produce the same period changes as a bump placed in the core. 
Table 10. Gaia data for all observed targets. We list the parallax (col 2), distance in pc (col 3), apparent $G$ magnitude (col 4) and colour (col 5), along with the absolute magnitude $M_{G}(\mathrm{col} 6)$ and the stellar mass (col 7) and effective temperature (col 8) computed in this work (see the text for details). The last column indicates the status of the object from this work. * Distances computed by taking the inverse of the parallax angle.

\begin{tabular}{|c|c|c|c|c|c|c|c|c|}
\hline Star & Parallax (mas) & Distance (pc) & $G$ & $G_{\mathrm{bp}}-G_{\mathrm{rp}}$ & $M_{G}$ & $\operatorname{Mass}\left(\mathrm{M}_{\odot}\right)$ & $T_{\text {eff }}$ & Class \\
\hline SDSS J092511.63+050932.6 & $24.663 \pm 0.061$ & $40.499 \pm 0.100$ & 15.271 & 0.054 & 12.231 & $0.7117 \pm 0.0061$ & $10831 \pm 58$ & known \\
\hline WD1345-0055 & $9.820 \pm 0.105$ & $101.552 \pm 1.092$ & 16.789 & -0.005 & 11.750 & $0.5881 \pm 0.0117$ & $11533 \pm 125$ & known \\
\hline HE $1429-037$ & $14.341 \pm 0.105$ & $69.598 \pm 0.514$ & 16.033 & 0.040 & 11.816 & $0.5602 \pm 0.0134$ & $10889 \pm 162$ & known \\
\hline SDSS J161218.08+083028.1 & $7.662 \pm 0.183$ & $130.512 \pm 3.118 *$ & 17.831 & -0.025 & 12.253 & $0.8173 \pm 0.0280$ & $12062 \pm 248$ & known \\
\hline GD 385 & $21.115 \pm 0.037$ & $47.295 \pm 0.082$ & 15.149 & 0.014 & 11.772 & $0.5751 \pm 0.0008$ & $11247 \pm 60$ & known \\
\hline SDSS J221458.37-002511.9 & $7.011 \pm 0.211$ & $142.270 \pm 4.309$ & 17.923 & 0.025 & 12.516 & $0.7177 \pm 0.0398$ & $11227 \pm 364$ & known \\
\hline SDSS J235040.72-005430.9 & $4.665 \pm 0.265$ & $214.023 \pm 12.373$ & 18.121 & 0.170 & 11.465 & $0.2998 \pm 0.0283$ & $9370 \pm 180$ & known \\
\hline SDSS J082804.63+094956.6 & $6.460 \pm 0.181$ & $154.307 \pm 4.355$ & 17.710 & 0.037 & 11.761 & $0.5310 \pm 0.0431$ & $11027 \pm 283$ & new \\
\hline SDSS J094929.09+101918.8 & $6.959 \pm 0.168$ & $143.235 \pm 3.485$ & 17.580 & 0.031 & 11.793 & $0.5686 \pm 0.0431$ & $11067 \pm 288$ & new \\
\hline GD 195 & $9.441 \pm 0.192$ & $105.672 \pm 2.168$ & 16.632 & 0.038 & 11.508 & $0.4459 \pm 0.0164$ & $10700 \pm 100$ & new \\
\hline L495-82 & $42.779 \pm 0.043$ & $23.375 \pm 0.024 *$ & 13.764 & 0.027 & 11.920 & $0.6158 \pm 0.0023$ & $11106 \pm 30$ & new \\
\hline SDSS J095703.09+080504.8 & $8.767 \pm 0.166$ & $113.749 \pm 2.172$ & 17.704 & 0.035 & 12.418 & $0.7943 \pm 0.0343$ & $11067 \pm 308$ & possible \\
\hline WD1454-0111 & $9.536 \pm 0.159$ & $104.870 \pm 1.749 *$ & 17.343 & -0.049 & 12.239 & $0.8337 \pm 0.0325$ & $12413 \pm 335$ & NOV \\
\hline SDSS J161005.7+030256.1 & $4.437 \pm 0.239$ & $225.093 \pm 12.452$ & 18.550 & 0.018 & 11.785 & $0.5850 \pm 0.0512$ & $11307 \pm 345$ & NOV \\
\hline SDSS J235932.80-033541.1 & $3.254 \pm 0.245$ & $306.690 \pm 23.747$ & 17.910 & -0.035 & 10.472 & $0.2470 \pm 0.0247$ & $11340 \pm 240$ & NOV \\
\hline
\end{tabular}

\section{USING GAIA DATA}

Using the data from the Gaia mission, we have additional information on the ZZ Cetis. From the distance and magnitudes we can estimate the stellar mass and effective temperature, independently from the spectroscopy. Using hydrogen-rich atmosphere models for Gaia magnitudes (see Kepler et al. 2019, for details) combined with mass-radius relation from Romero et al. (2019), we transform absolute magnitude $M_{G}$ and colour $G_{\mathrm{bp}}-G_{\text {rp }}$ into stellar mass and effective temperature. The absolute magnitude is computed from the apparent magnitude and the distance. For stellar masses lower than $0.5 \mathrm{M}_{\odot}$ we use the atmosphere models from the Montreal Group (Bergeron, private communication) (see also Bergeron et al. 2011). Note that the uncertainties in the effective temperature are underestimated since the magnitude filter from the Gaia satellite are quite broad, in the case of white dwarf stars.

The results are summarized in Table 10. We list parallax, distance, $G$ apparent magnitude, and colour $G_{\mathrm{bp}}-G_{\mathrm{rp}}$ in columns 2, 3, 4, and 5, respectively. The distance was taken from Bailer-Jones et al. (2018), except for the objects marked with an asterisk, for which we compute the distance from the inverse of the parallax. Since for all objects the uncertainties in the parallax is less than 5 percent, we do not expect large deviations (Bailer-Jones et al. 2018). Also listed are the absolute magnitude $M_{G}(\mathrm{col} 6)$ and the stellar mass (col 7) and effective temperature (col 8) computed in this work. In the last column, we specify the status of the star, as known variable, new variable, possible variable, and NOV.

We compare the stellar mass obtained from distance and Gaia magnitudes (Table 10) with the determinations obtained from spectroscopic values of $\log g$ and effective temperature (Table 1) and the seismological mass (Table 9). Since the evolutionary models used to obtain a seismological representative model for each object are the same that we used to derive the spectroscopic mass from

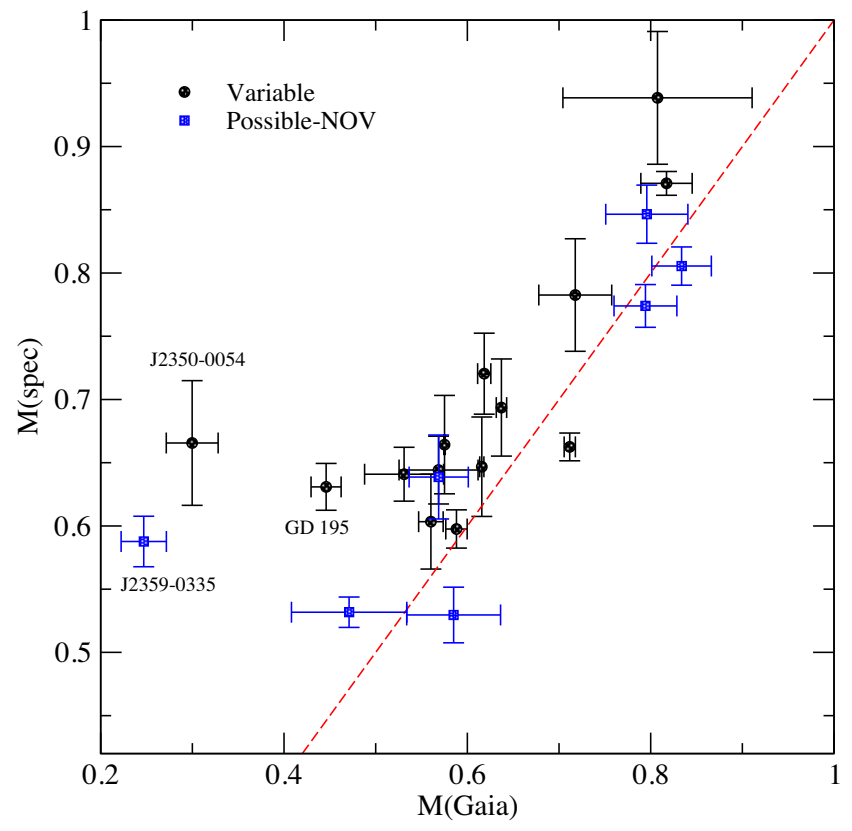

Figure 9. Comparison between the values of the stellar mass according to Gaia data and spectroscopy for all observed targets. Variable white dwarf stars, new and known variables, are depicted with the black circles, while the objects not classified as variables, possible and NOV, are depicted with the blue squares. The uncertainties are the internal uncertainties of the fitting procedure. The red line represents the 1:1 correspondence.

the observed spectroscopic parameters and to determine the massradius relation for the atmosphere models, this comparison is worth doing. The results are depicted in Figs 9 and 10. 


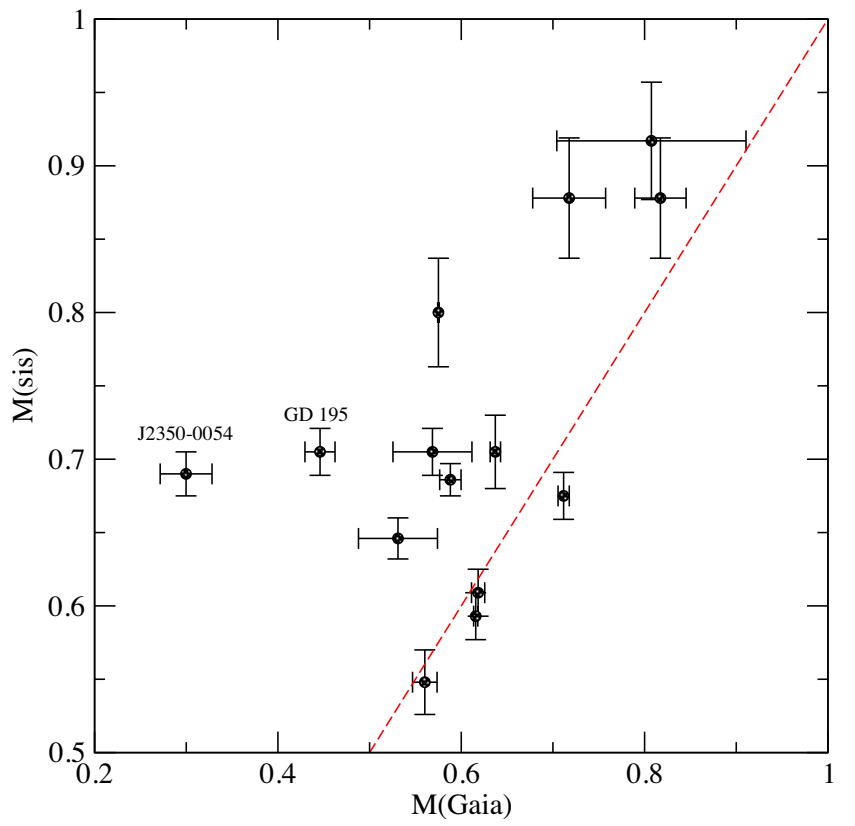

Figure 10. Comparison between the values of the stellar mass according to Gaia data and asteroseismology for all observed targets showing confirmed photometric variability. The uncertainties are the internal uncertainties of the fitting procedure. The red line represents the 1:1 correspondence.

The comparison between the stellar mass based on Gaia data and spectroscopy is presented in Fig. 9. The variable DA white dwarfs are depicted with the black circles, while the objects with no confirmed variability are depicted with the blue squares. The uncertainties are the internal uncertainties of the fitting procedure. For most objects, the correspondence between both determinations is not in good agreement, specially for three objects: SDSS J235040.72-005430.9, GD 195, and SDSS J235932.80-035541.1. In these cases, the stellar mass based on Gaia data is that of a low-mass white dwarf, with stellar masses of 0.2998, 0.4459, and $0.2470 \mathrm{M}_{\odot}$, respectively (see Table 10 for details). In particular, SDSS J235040.72-005430.9 has been a mystery since the discovery of its variability by Mukadam et al. (2004), showing a spectroscopic temperature characteristic of the red edge and short pulsation periods, characteristic of the blue edge of the instability strip. As was mentioned in Section 5.2, this object can indeed be part of a WD+WD binary system, where the flux is dominated by the less-massive brighter component (Fuchs 2018). Given this evidence, it is possible that GD 195, and specially SDSS J235932.80-035541.1 are also part of an unresolved double degenerate binary system.

A similar trend is found when we compare the stellar mass based in Gaia data and the seismological mass obtained from our fits. Since SDSS J235932.80-035541.1 is classified as NOV it is not depicted in this figure. As expected, SDSS J235932.80-035541.1 lays above the $1: 1$ correspondence line, with a seismological mass of $0.69 \mathrm{M}_{\odot}$. The same happens for GD 195, with a seismological mass of $0.705 \mathrm{M}_{\odot}$.

\section{ANALYSIS OF THE SAMPLE}

In this section we analyse the main results of a sample of $\sim 91$ ZZ Cetis with asteroseismological fits. We include the results from

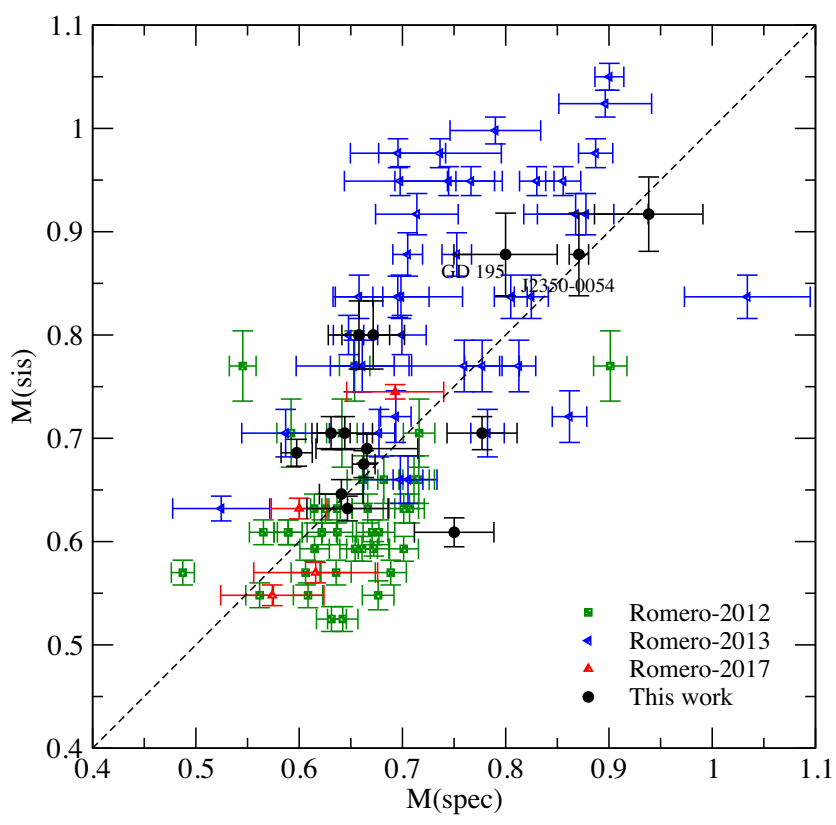

Figure 11. Comparison between the values of the stellar mass obtained from spectroscopy with 3D convection correction and asteroseismology for a sample of $91 \mathrm{ZZ}$ Ceti stars. The sample is taken from Romero et al. (2012) (green squares), Romero et al. (2013) (blue right-triangle), Romero et al. (2017) (red up-triangle), and this work (black circle). The uncertainties are the internal uncertainties of the fitting procedure. The dashed line represents the $1: 1$ correspondence.

previous asteroseismological fits that used the same grid of models, to be consistent with the results obtained in this work. From the works of Romero et al. (2012, 2013) and Romero et al. (2017) we selected 77 objects. Finally, we include the 14 ZZ Cetis analysed in this work, with 10 previously known variables and the four new ZZ Cetis. In case one object was analysed more than once, we choose the asteroseismological solution from the most recent asteroseismological fit.

In Fig. 11 we compare the stellar mass obtained from spectroscopy and seismology for the sample of $91 \mathrm{ZZ}$ Cetis. The spectroscopic mass is taken from Table 1, with 3D convection correction. The general agreement between both sets of estimates is not quite good, the largest discrepancy being for stellar masses above $\sim 0.75 \mathrm{M}_{\odot}$. Note that $3 \mathrm{D}$ convection correction in $\log g$ is not completely efficient in the high-mass regime (Tremblay et al. 2019), and thus could be the reason for the deviation seen in that mass range. However, the bulk of point in Fig. 11 accumulates around the 1:1 correspondence line, demonstrating that no appreciable offset exists between the spectroscopic and asteroseismic estimations of the stellar mass. The mean spectroscopic mass for the sample of 91 ZZ Cetis is $\left\langle M_{\text {spec }}\right\rangle=0.692 \mathrm{M}_{\odot}, \sim 5$ per cent lower than the mean seismological mass for the same sample $\left\langle M_{\text {sis }}\right\rangle=0.727 \mathrm{M}_{\odot}$. Note that these values are largely affected by the 36 massive ZZ Cetis analysed by Romero et al. (2013), with stellar masses larger than $0.72 \mathrm{M}_{\odot}$, affected by the possible shortcoming in the 3D convection correction. Thus, these sample should not be compared with other samples with a homogeneous distribution in stellar mass. If we do not consider the sample from Romero et al. (2013), we obtain an average spectroscopic mass of $\left\langle M_{\text {spec }}\right\rangle=0.657 \mathrm{M}_{\odot}$, which is only 1.7 per cent higher than the corresponding mean seismological mass of $\left\langle M_{\text {sis }}\right\rangle=0.646 \mathrm{M}_{\odot}$. 


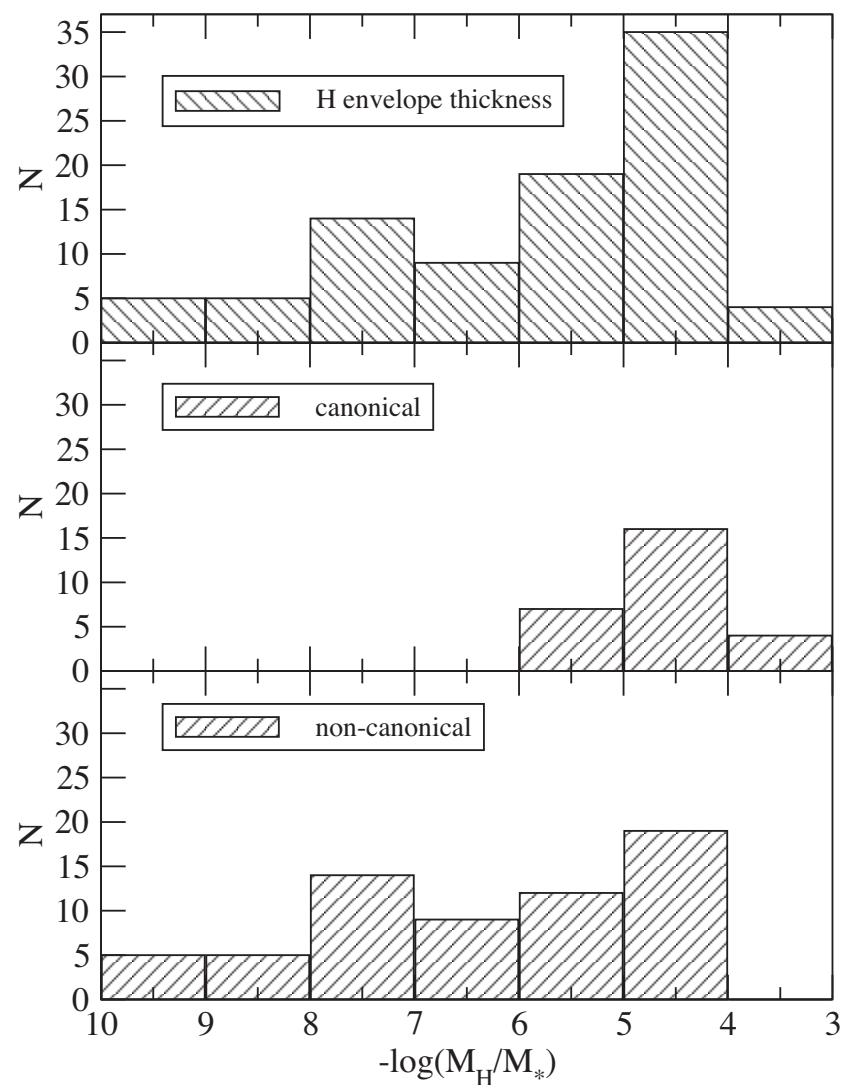

Figure 12. Upper panel: histogram showing the hydrogen envelope thickness distribution for the sample of 77 ZZ Cetis stars. Middle panel: histogram for models with canonical hydrogen envelope thickness, as predicted by canonical evolutionary computations according to the value of the stellar mass. Lower panel: histogram for models with non-canonical envelope thickness.

One of the parameters that can be estimated almost exclusively by asteroseismology is the hydrogen mass left in the envelope of a DA white dwarf star. The value of the hydrogen envelope mass for the objects analysed in this work is listed in column 5 of Table 9. Note that, depending on the stellar mass, the canonical hydrogen envelopes can have masses ranging from $\sim 10^{-3} M_{*}$, for $0.49 \mathrm{M}_{\odot}$ to $10^{-6} M_{*}$ for $\sim 1 \mathrm{M}_{\odot}$ (Romero et al. 2012, 2019).

Fig. 12 shows the distribution of the hydrogen envelope thickness for a sample of $91 \mathrm{ZZ}$ Ceti stars (upper panel), taken from Romero et al. $(2012,2013,2017)$ and this work. The middle and bottom panels show the distribution for the canonical envelopes, those with the thickest envelope allowed by single stellar evolution, and the thin envelopes, respectively.

From the distribution of hydrogen envelope mass, we note a pronounced maximum of the distribution for $\log \left(M_{\mathrm{H}} / M_{*}\right)$ in the range -5 to -4 , with contributions from both thin envelopes, for the low-mass models, and canonical envelopes for masses above $\sim 0.60 \mathrm{M}_{\odot}$. A second peak for $\log \left(M_{\mathrm{H}} / M_{*}\right)$ between -7 and -8 is also present in the distribution, with contributions mainly from the high-mass ZZ Cetis (Romero et al. 2013). From our sample of 91 ZZ Cetis, we found that 35 per cent of the best-fitting models have canonical envelopes, those with the thickest envelope as predicted by single stellar evolution. However, as much as 75 per cent show hydrogen envelopes thicker than $10^{-6} M_{*}$ and only 13 per cent shows very thin hydrogen envelopes with masses below $10^{-8} M_{*}$. This result is in agreement with the results presented by Clemens et al. (2017) from a sample of 16 hot ZZ Ceti stars. They found that the best-matching models, taken from the model grid presented in Romero et al. (2012), have hydrogen layer masses values at or near the canonically thick limit calculated from nuclear burning, which is consistent with our results.

The mean value of the hydrogen layer mass is $\left\langle M_{\mathrm{H}} / M_{*}\right\rangle=$ $2.3 \times 10^{-6}$. This value is approximately five times larger than that obtained by Castanheira \& Kepler (2009), with a sample covering a broad range in stellar mass, and using a different model grid. In spite of this difference, both studies conclude that the possible values for the hydrogen envelope span over a large range $\left(10^{-4}-10^{-10} M_{*}\right)$, with a fraction of DA white dwarf stars formed with a hydrogen envelope much thinner than that predicted by single stellar evolution computations. An excellent example of a DA white dwarf with a measured thin hydrogen envelope is 40 Eridani B. Romero et al. (2019) obtained a hydrogen mass of $M_{\mathrm{H}}=2.6 \times 10^{-8} \mathrm{M}_{\odot}$ by comparing the theoretical massradius relations for different hydrogen envelope masses with the dynamical stellar mass from Mason et al. (2017) and the radius obtained from photometry and distance (Bond, Bergeron \& Bédard 2017).

Another evidence of the existence of DA white dwarf with thin hydrogen envelopes was presented by Ourique et al. (2019), who studied the spectral evolution of white dwarf stars, using a sample of $\sim 13000$ DA and 3000 non-DA white dwarf stars with both spectroscopic data from SDSS DR12 catalogue and the Gaia DR2 survey. The authors found that the ratio of non-DA to DA white dwarfs is $\sim 0.075$ for effective temperatures above $22000 \mathrm{~K}$ and increases by a factor of five for effective temperatures cooler than $15000 \mathrm{~K}$. The most likely explanation for the spectral evolution is the convective mixing of a thin hydrogen envelope into the underlying helium layer of $14 \pm 3$ percent of DA white dwarf stars.

\section{CONCLUSIONS}

In this work we present the results from ground-based observations applied to the search of variable DA white dwarf stars. We report the discovery of four new variables: SDSS J082804.63+094956.6, SDSS J094929.09+101918.8, GD 195, and L495-82. In addition we re-observed 10 known ZZ Cetis to look for new periodicities and to study the stability of the pulsation periods. From the sample of 12 candidates, four objects are classified as possible variables, with peaks in the FT with amplitudes above $3 \sigma$ but below $4 \sigma$, the latter being the detection limit adopted in this work. Our main results are listed below.

The candidates were selected from the SDSS white dwarf catalogue (e.g. Kepler et al. 2019) complemented by the list of DA white dwarfs presented in Bédard et al. (2017). Using the sample of known ZZ Cetis, we selected those objects with spectroscopic atmospheric parameters within the empirical instability strip. Since we found new variables among our candidates we believe that this selection method is adequate. Currently, we have $\sim 570$ candidates from the SDSS white dwarf catalogue (Kepler et al. 2019) within the instability strip that have not been studied for variability.

By comparing stellar mass determinations from spectroscopy and seismology with that obtained using Gaia data, we found three outliers. The stellar mass of SDSS J235040.72-005430.9, 
SDSS J235932.8-033541.1, and GD 195 determined using photometry and parallax from Gaia is $0.299,0.247$, and $0.4459 \mathrm{M}_{\odot}$, respectively, below the stellar mass obtained with spectroscopy, and incompatible with single stellar evolution. Since the lowest mass considered in our model grid is $0.493 \mathrm{M}_{\odot}$, the seismological mass for SDSS J235040.72-005430.9 and GD 195 is also higher than that obtained with Gaia. In particular, there is evidence that SDSS J235040.72-005430.9 could be an unresolved WD+WD system (Fuchs 2018), with the flux dominated by the less-massive brighter companion. Thus, within this hypothesis, it is possible that GD 195 and specially SDSS J235932.8-033541.1 are also an unresolved double degenerate system.

Finally, we analyse the properties of a sample of 91 ZZ Ceti stars that were subject of an asteroseismological study. The distribution of hydrogen envelope mass spans the range $-\log \left(M_{\mathrm{H}} / M_{*}\right)=4-10$, with a pronounced maximum for $\log \left(M_{\mathrm{H}} / M_{*}\right)$ between -4 and -5 , in agreement with the results obtained by Clemens et al. (2017) based solely on observational data. The mean value for our sample is $\left\langle M_{\mathrm{H}} / M_{*}\right\rangle=2.3 \times 10^{-6}$. Note that 91 objects correspond to $\sim 36$ per cent of all the ZZ Cetis known to date.

\section{ACKNOWLEDGEMENTS}

ADR, LAA, GO, GRL, and SOK acknowledge financial support from CNPq and PRONEX-FAPERGS/CNPq (Brazil). TK acknowledges financial support from Capes (Brazil). DS is supported by the Gemini Observatory, which is operated by the Association of Universities for Research in Astronomy, Inc., on behalf of the international Gemini partnership of Argentina, Brazil, Canada, Chile, the Republic of Korea, and the United States of America. IP acknowledges funding by the Deutsche Forschungsgemeinschaft under grant GE2506/12-1.

Based on observations obtained at Observatório do Pico dos Dias/LNA, at the Southern Astrophysical Research (SOAR) telescope, which is a joint project of the Ministério da Ciência, Tecnologia, Inovação e Comunicaçẽs (MCTIC) do Brasil, the US National Optical Astronomy Observatory (NOAO), the University of North Carolina at Chapel Hill (UNC), and Michigan State University (MSU) and processed using the Gemini IRAF package, which is operated by the Association of Universities for Research in Astronomy, Inc., under a cooperative agreement with the NSF on behalf of the Gemini partnership: the National Science Foundation (United States), the National Research Council (Canada), CONICYT (Chile), Ministerio de Ciencia, Tecnología e Innovación Productiva (Argentina) and Ministério da Ciência, Tecnologia, Inovação e Comunicaçẽs (Brasil). This work has used data from the European Space Agency (ESA) mission Gaia (https://www.cosmos.esa.int/gaia), processed by the Gaia Data Processing and Analysis Consortium (DPAC, https://www.cosm os.esa.int/web/gaia/dpac/consortium). Funding for the DPAC has been provided by national institutions, in particular the institutions participating in the Gaia Multilateral Agreement. This research has also made use of the TOPCAT (http://www.starlink.ac.uk/topcat/) software (Taylor 2005), The Montreal White Dwarf Database (Dufour et al. 2017, http://www.montrealwhitedwarfdatabase.org /home.html), and NASA Astrophysics Data System. We thank P. Bergeron for sharing the atmosphere models for DA white dwarf for Gaia filters. The observational data are available upon request to alejandra.romero@ufrgs.br.

\section{REFERENCES}

Althaus L. G., Córsico A. H., Bischoff-Kim A., Romero A. D., Renedo I., García-Berro E., Miller Bertolami M. M., 2010, ApJ, 717, 897

Bailer-Jones C. A. L., Rybizki J., Fouesneau M., Mantelet G., Andrae R., 2018, AJ, 156, 58

Baran A. S., 2013, Acta Astron., 63, 203

Bédard A., Bergeron P., Fontaine G., 2017, ApJ, 848, 11

Bell K. J., Hermes J. J., Bischoff-Kim A., Moorhead S., Montgomery M. H., Østensen R., Castanheira B. G., Winget D. E., 2015, ApJ, 809, 14

Bell K. J., Hermes J. J., Montgomery M. H., Winget D. E., Gentile Fusillo N. P., Raddi R., Gänsicke B. T., 2017, in Tremblay P.-E., Gaensicke B., Marsh T., eds, ASP Conf. Ser.Vol. 509, 20th European White Dwarf Workshop. Astron. Soc. Pac., San Francisco, p. 303

Bell K. J. et al., 2019, Res. Notes Am. Astron. Soc., 3, 81

Bergeron P. et al., 2011, ApJ, 737, 28

Bognar Z., Sodor A., 2016, Inf. Bull. Var. Stars, 6184, 1

Bond H. E., Bergeron P., Bédard A., 2017, ApJ, 848, 16

Brickhill A. J., 1991, MNRAS, 251, 673

Castanheira B. G., Kepler S. O., 2008, MNRAS, 385, 430

Castanheira B. G., Kepler S. O., 2009, MNRAS, 396, 1709

Castanheira B. G. et al., 2006, A\&A, 450, 227

Castanheira B. G. et al., 2007, A\&A, 462, 989

Castanheira B. G., Kepler S. O., Kleinman S. J., Nitta A., Fraga L., 2010, MNRAS, 405, 2561

Castanheira B. G., Kepler S. O., Kleinman S. J., Nitta A., Fraga L., 2013, MNRAS, 430, 50

Clemens J. C., 1993, Balt. Astron., 2, 407

Clemens J. C., O’Brien P. C., Dunlap B. H., Hermes J. J., 2017, in Tremblay P.-E., Gaensicke B., Marsh T., eds, ASP Conf. Ser.Vol. 509, 20th European White Dwarf Workshop. Astron. Soc. Pac., San Francisco, p. 255

Córsico A. H., Althaus L. G., 2006, A\&A, 454, 863

Córsico A. H., Althaus L. G., Miller Bertolami M. M., Kepler S. O., 2019, A\&AR, 27, 7

Dolez N., Vauclair G., 1981, A\&A, 102, 375

Dufour P., Blouin S., Coutu S., Fortin-Archambault M., Thibeault C., Bergeron P., Fontaine G., 2017, in Tremblay P.-E., Gaensicke B., Marsh T., eds, ASP Conf. Ser.Vol. 509, 20th European Workshop on White Dwarfs . Astron. Soc. Pac., San Francisco, p. 3

Fontaine G., Brassard P., 2008, PASP, 120, 1043

Gianninas A., Bergeron P., Fontaine G., 2006, AJ, 132, 831

Gianninas A., Bergeron P., Ruiz M. T., 2011, ApJ, 743, 138

Gilliland R. L. et al., 2010, ApJ, 713, L160

Goldreich P., Wu Y., 1999, ApJ, 511, 904

Hermes J. J. et al., 2014, ApJ, 789, 85

Hermes J. J. et al., 2017, ApJS, 232, 23

Hesser J. E., Lasker B. M., Neupert H. E., 1976, ApJ, 209, 853

Josh F., 2018, Presentation in the 21st European White Dwarf Workshop. Austin, Texas

Kepler S. O., 1993, Balt. Astron., 2, 515

Kepler S. O., Romero A. D., 2017, in EPJ Web Conf., 01011, EPJWC.152

Kepler S. O. et al., 2005, A\&A, 442, 629

Kepler S. O. et al., 2012, ApJ, 757, 177

Kepler S. O. et al., 2016, MNRAS, 455, 3413

Kepler S. O. et al., 2019, MNRAS, 486, 2169

Kleinman S. J. et al., 2013, ApJS, 204, 5

Koester D., 2010, Mem. Soc. Astron. Ital., 81, 921

Lenz P., Breger M., 2004, in Zverko J., Ziznovsky, J., Adelman S. J., Weiss W. W., eds, Proc. IAU Symp. 224, The A-Star Puzzle. Cambridge University Press, Cambridge, p. 786

Mason B. D., Hartkopf W. I., Miles K. N., 2017, AJ, 154, 200

Montgomery M. H., Metcalfe T. S., Winget D. E., 2003, MNRAS, 344, 657

Montgomery M. H. et al., 2010, ApJ, 716, 84

Mukadam A. S. et al., 2004, ApJ, 607, 982

Mukadam A. S., Montgomery M. H., Winget D. E., Kepler S. O., Clemens J. C., 2006, ApJ, 640, 956 
Mullally F., Thompson S. E., Castanheira B. G., Winget D. E., Kepler S. O., Eisenstein D. J., Kleinman S. J., Nitta A., 2005, ApJ, 625, 966

Ourique G., Romero A. D., Kepler S. O., Koester D., Amaral L. A., 2019, MNRAS, 482, 649

Renedo I., Althaus L. G., Miller Bertolami M. M., Romero A. D., Córsico A. H., Rohrmann R. D., García-Berro E., 2010, ApJ, 717, 183

Ricker G. R. et al., 2014, in Oschmann J. M. Jr., Clampin M., Fazio G. G., MacEwen H. A., eds, Proc. SPIE Conf. Ser.Vol. 9143, Space Telescopes and Instrumentation 2014: Optical, Infrared, and Millimeter Wave. SPIE, Bellingham, p. 914320

Romero A. D., Córsico A. H., Althaus L. G., Kepler S. O., Castanheira B. G., Miller Bertolami M. M., 2012, MNRAS, 420, 1462

Romero A. D., Kepler S. O., Córsico A. H., Althaus L. G., Fraga L., 2013, ApJ, 779, 58

Romero A. D., Campos F., Kepler S. O., 2015, MNRAS, 450, 3708

Romero A. D. et al., 2017, ApJ, 851, 60

Romero A. D., Kepler S. O., Joyce S. R. G., Lauffer G. R., Córsico A. H., 2019, MNRAS, 484, 2711

Rowan D. M., Tucker M. A., Shappee B. J., Hermes J. J., 2019, MNRAS, 486,4574
Silvotti R., Voss B., Bruni I., Koester D., Reimers D., Napiwotzki R., Homeier D., 2005, A\&A, 443, 195

Su J., Fu J., Lin G., Chen F., Khokhuntod P., Li C., 2017, ApJ, 847, 34

Taylor M. B., 2005, in Shopbell P., Britton M., Ebert R., eds, ASP Conf. Ser. Vol. 347, Astronomical Data Analysis Software and Systems XIV. Astron. Soc. Pac., San Francisco, p. 29

Tremblay P.-E., Ludwig H.-G., Steffen M., Freytag B., 2013, A\&A, 552, A13

Tremblay P.-E., Cukanovaite E., Gentile Fusillo N. P., Cunningham T., Hollands M. A., 2019, MNRAS, 482, 5222

Voss B., Koester D., Østensen R., Kepler S. O., Napiwotzki R., Homeier D., Reimers D., 2006, A\&A, 450, 1061

Winget D. E., van Horn H. M., Tassoul M., Fontaine G., Hansen C. J., Carroll B. W., 1982, ApJ, 252, L65

Zhang E.-H., Robinson E. L., Nather R. E., 1986, ApJ, 305, 740

\section{APPENDIX}


Table A1. Uncertainties in the measured frequencies and their amplitudes.

\begin{tabular}{|c|c|c|c|c|c|}
\hline Known & $\begin{array}{c}\text { Freq } \\
(\mu \mathrm{Hz})\end{array}$ & $\begin{array}{c}\sigma_{\text {freq }} \\
(\mu \mathrm{Hz})\end{array}$ & $\begin{array}{c}\text { Amp } \\
(\mathrm{mma})\end{array}$ & $\begin{array}{c}\sigma_{\mathrm{amp}} \\
(\mathrm{mma})\end{array}$ & $\begin{array}{l}\text { Period } \\
\text { (s) }\end{array}$ \\
\hline \multirow[t]{6}{*}{ BPM 30551} & 12033.238 & 0.02 & 11.2 & 0.7 & 831.031 \\
\hline & 1289.930 & 5 & 10.2 & 0.7 & 775.235 \\
\hline & 1041.895 & 0.02 & 7.7 & 0.6 & 959.789 \\
\hline & 2173.602 & 0.02 & 5.7 & 0.5 & 460.065 \\
\hline & 1013.831 & 0.08 & 5.5 & 1 & 986.357 \\
\hline & 1540.005 & 0.03 & 5.5 & 0.5 & 649.348 \\
\hline SDSS J092511.63+050932.6 & 801.628 & 13 & 8 & 1 & 1247.5 \\
\hline HS $1249+0426$ & 3391.095 & 9 & 14.5 & 3 & 294.91 \\
\hline WD1345-0055 & 5121.860 & 84 & 8.9 & 3 & 195.24 \\
\hline HE 1429-037 & 1216.918 & 35 & 56.93 & 15 & 821.74 \\
\hline \multirow[t]{2}{*}{ GD 385} & 3904.829 & 4 & 9.4 & 0.5 & 256.09 \\
\hline & 7816.344 & 35 & 3.5 & 0.8 & 127.9 \\
\hline \multirow[t]{2}{*}{ SDSS J215905.53+132255.8 } & 1339.282 & 5 & 24.2 & 1 & 746.7 \\
\hline & 1473.210 & 47 & 8 & 2 & 678.8 \\
\hline SDSS J221458.37-002511.9 & 3920.273 & 4 & 16 & 2 & 255.1 \\
\hline \multirow[t]{3}{*}{ SDSS J235040.72-005430.9 } & 3281.518 & 7 & 18.29 & 2 & 304.7 \\
\hline & 2562.004 & 12 & 10.17 & 2 & 390.3 \\
\hline & 3678.258 & 57 & 8.2 & 3 & 271.9 \\
\hline \multicolumn{6}{|l|}{ New } \\
\hline \multirow[t]{3}{*}{ SDSS J08204.638+094956.6 } & 3499.073 & 0.4 & 14.2 & 0.8 & 285.79 \\
\hline & 5093.984 & 0.4 & 10.9 & 0.8 & 196.31 \\
\hline & 3909.61 & 0.8 & 5.7 & 0.6 & 255.78 \\
\hline \multirow[t]{3}{*}{ SDSS J094929.09+101918.8 } & 5017.309 & 0.08 & 3.3 & 0.4 & 199.31 \\
\hline & 3434.066 & 0.02 & 1.9 & 0.5 & 291.2 \\
\hline & 8403.361 & 0.01 & 2 & 0.4 & 119 \\
\hline \multirow[t]{2}{*}{ GD 195} & 2149.798 & 0.2 & 8.7 & 0.1 & 465.16 \\
\hline & 1540.375 & 0.2 & 6.2 & 0.1 & 649.2 \\
\hline \multirow[t]{12}{*}{ L495-82 } & 1108.125 & 13 & 10.48 & 3 & 902.425 \\
\hline & 908.856 & 21 & 6.72 & 3 & 1100.283 \\
\hline & 1244.21 & 30 & 5.74 & 3 & 803.722 \\
\hline & 1404.597 & 10 & 3.92 & 4 & 711.947 \\
\hline & 2746.011 & 21 & 3.59 & 4 & 364.164 \\
\hline & 991.48 & 51 & 3.74 & 2 & 1008.59 \\
\hline & 1720.509 & 38 & 2.62 & 1 & 581.223 \\
\hline & 2396.375 & 14 & 2.001 & 0.3 & 417.296 \\
\hline & 4578.305 & 17 & 1.41 & 0.3 & 218.421 \\
\hline & 2031.562 & 181 & 1.39 & 3 & 492.23 \\
\hline & 3018.182 & 121 & 1.21 & 9 & 331.32 \\
\hline & 2541.883 & 295 & 1.19 & 5 & 393.409 \\
\hline \multicolumn{6}{|l|}{ Candidate } \\
\hline \multirow[t]{2}{*}{ SDSS J095703.09+080504.8 } & 8321.928 & 0.4 & 12.9 & 3 & 120.2 \\
\hline & 13850.564 & 2 & 11.7 & 3 & 72.2 \\
\hline LP $375-51$ & 909.750 & 303 & 3.6 & 2 & 1099.2 \\
\hline \multirow[t]{3}{*}{ SDSS J212441.27-073234.9 } & 9218.58 & 0.6 & 6.9 & 2 & 108.5 \\
\hline & 4108.62 & 0.7 & 5.9 & 1 & 243.4 \\
\hline & 7438.69 & 15 & 5.9 & 2 & 134.4 \\
\hline \multirow[t]{2}{*}{ SDSS J213159.88+010856.3 } & 3281.458 & 25 & 11.11 & 4 & 304.7 \\
\hline & 11095.163 & 22 & 10.5 & 3 & 90.1 \\
\hline
\end{tabular}

This paper has been typeset from a $\mathrm{T}_{\mathrm{E}} \mathrm{X} / \mathrm{L} \mathrm{T} \mathrm{E}$ file prepared by the author. 